\title{
Linear instability of planar shear banded flow of both diffusive and non-diffusive Johnson-Segalman fluids
}

\author{
Helen J. Wilson ${ }^{\mathrm{a}, *}$ and Suzanne M. Fielding ${ }^{\mathrm{b}}$ \\ ${ }^{a}$ Department of Mathematics, University College London, Gower Street, London \\ WC1E 6BT, UK \\ ${ }^{\mathrm{b}}$ School of Mathematics, Lamb Building, The University of Manchester, Booth \\ Street East, Manchester M13 9EP, UK
}

\begin{abstract}
We consider the linear stability of shear banded planar Couette flow of the JohnsonSegalman fluid, with and without the addition of stress diffusion to regularise the equations. In particular, we investigate the linear stability of an initially onedimensional "base" flow, with a flat interface between the bands, to two-dimensional perturbations representing undulations along the interface. We demonstrate analytically that, for the linear stability problem, the limit in which diffusion tends to zero is mathematically equivalent to a pure (non-diffusive) Johnson-Segalman model with a material interface between the shear bands, provided the wavelength of perturbations being considered is long relative to the (short) diffusion lengthscale.

For no diffusion, we find that the flow is unstable to long waves for almost all arrangements of the two shear bands. In particular, for any set of fluid parameters and shear stress there is some arrangement of shear bands that shows this instability. Typically the stable arrangements of bands are those in which one of the two bands is very thin. Weak diffusion provides a small stabilising effect, rendering extremely long waves marginally stable. However, the basic long-wave instability mechanism is not affected by this, and where there would be instability as wavenumber $k \rightarrow 0$ in the absence of diffusion, we observe instability for moderate to long waves even with diffusion.
\end{abstract}

This paper is the first full analytical investigation into an instability first documented in the numerical study of [1]. Authors prior to that work have either happened to choose parameters where long waves are stable or used slightly different constitutive equations and Poiseuille flow, for which the parameters for instability appear to be much more restricted.

We identify two driving terms that can cause instability: one, a jump in $N_{1}$, as reported previously by Hinch et al. [2]; and the second, a discontinuity in shear rate. The mechanism for instability from the second of these is not thoroughly understood. 
We discuss the relevance of this work to recent experimental observations of complex dynamics seen in shear-banded flows.

Key words: shear-banding fluid, linear instability, Couette flow, diffusive Johnson-Segalman fluid, interfacial instability

PACS: 47.50.+d Non-Newtonian fluid flows, 47.20.-k Hydrodynamic stability, 83.60.Wc Rheology: flow instabilities

\section{Introduction}

Complex fluids such as wormlike [3] and onion [4] surfactants commonly show flow instabilities and flow-induced transitions that lead to spatially heterogeneous, "shear banded" states. In shear thinning wormlike micelles, for example, homogeneous flow becomes unstable above a critical shear rate. The system then separates into bands of differing viscosity and internal structure, separated by an interface that has its normal in the flow-gradient direction. Widespread experimental observations of this phenomenon have been made by flow birefringence [5], and by NMR [6] and ultrasound velocity imaging [7]. More recently, fluidity banding has been reported in soft glassy materials [8]. In bulk mechanical measurements, the main signature of shear banding is a kink followed by a plateau in the steady state flow curve.

Beyond this basic picture, an accumulating body of data reveals that shear banded states can fluctuate. Early evidence came from unsteady erratic [9] or periodic $[10,11]$ fluctuations in the wall stress at an applied value of the shear rate. More recent velocimetry experiments with enhanced spatial and temporal resolution have unambiguously revealed fluctuations in the interface between the bands $[7,12-15]$. To date, however, most theoretical studies have considered only a flat, stationary interface. In this paper, therefore, we study analytically the linear instability of shear banded flow with respect to small undulations along the interface.

Theoretically, shear banding is thought to arise from a non-monotonicity in the underlying constitutive relation between the shear stress and shear rate for homogeneous flow $[16,17,6,18]$. The simplest constitutive model to mimic this dependence (apart from "toy" models that do not obey the principle of material frame indifference) is the Johnson-Segalman (JS) model [19]. A sample plot of shear stress against shear rate in simple shear flow for this fluid

\footnotetext{
* Corresponding author

Email addresses: helen.wilson@ucl.ac.uk (Helen J. Wilson), suzanne.fielding@manchester.ac.uk (Suzanne M. Fielding).
} 


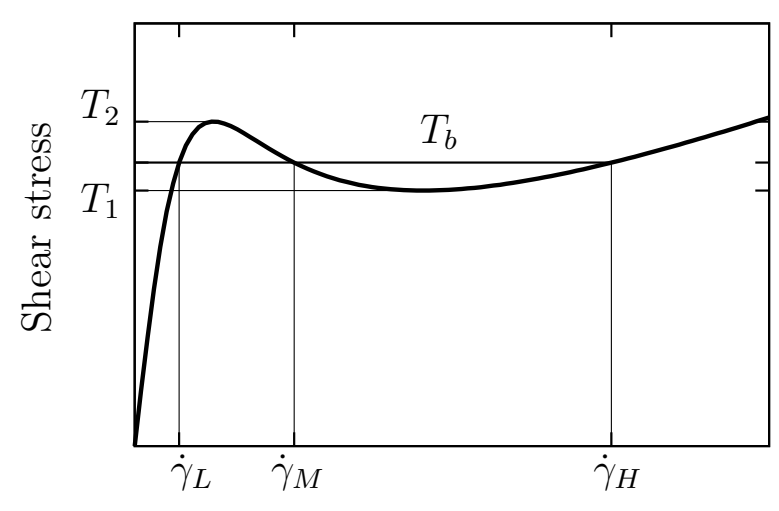

Shear rate

Fig. 1. A typical plot of shear stress against shear rate for steady, homogeneous shear flow of a shear-banding fluid. Here we use the Johnson-Segalman model with parameters $\epsilon=0.05, a=0.8$. If the shear stress is $T_{b}$, homogeneous shear flow with any of the shear rates $\dot{\gamma}_{L}<\dot{\gamma}_{M}<\dot{\gamma}_{H}$ is permitted; but flow with shear rate $\dot{\gamma}_{M}$ is unstable to one-dimensional perturbations.

is given in figure 1. Homogeneous flow with a shear rate on the decreasing part of the curve (e.g. $\dot{\gamma}_{M}$ ) is unstable to one-dimensional perturbations with wavevector in the flow-gradient direction [20]. The fluid therefore separates into a structure comprising bands of differing shear rates $\dot{\gamma}_{L}$ and $\dot{\gamma}_{H}$, one on each of the stable, upward-sloping parts of the curve. The interface between the bands has its normal in the flow-gradient direction. The shear stress $T$ is uniform across the whole flow, as required by a force balance.

The JS model in its original form contains no mechanism for uniquely selecting the shear stress $T_{b}$ at which banding occurs. Instead, in a numerical study of shear banding in such a model, the shear stress in the steady banded state depends strongly on the startup history [21-24], and can have a stress anywhere in the range $T_{1}<T_{b}<T_{2}$ in figure 1 . This conflicts notably with experiment, which consistently reveals a highly reproducible banding stress.

It is therefore critical to regularise the model in some way, to ensure stress selection. This is achieved by modifying the constitutive equation to include a diffusive ("non-local") term [22,25-27]. This mechanism was first proposed in 1989 by El-Kareh \& Leal [28], with the physical interpretation that individual polymer molecules can slowly diffuse across the interface, carrying their stress histories with them. Such terms also arise naturally in models of liquid crystalline dynamics. Regardless of their physical origin, non local terms lead to the selection of a unique, reproducible shear banding stress, $T_{b}$, as seen experimentally. They also provide a length scale for the thickness of the interface. In contrast, in the local model the interface is unphysically sharp: the flow variables jump discontinuously across it.

The exact details of how stress diffusion should be added to the JS model vary 
from author to author. The most commonly used version is due to Olmsted \& coworkers [29,30], in which a term proportional to the Laplacian of the extra stress, $\nabla^{2} \underline{\underline{\Sigma}}$, is added to the evolution equation for the polymeric stress. In this paper we will use a slightly more general form that is also capable of incorporating the model introduced by Yuan [31], in which the term added is a negative multiple of the Laplacian of the rate of strain.

The theoretical framework just described has been developed largely in the context of one dimensional (1D) studies that consider only the flow gradient direction, normal to the interface between the bands [21,32,33]. Clearly, such studies assume from the outset that the interface between the bands is perfectly flat and they predict (with few exceptions: $[34,35]$ ) time-independent banded states. This is clearly at odds with the accumulating body of data described above, revealing fluctuations of the banded state.

In view of this, a crucial question is whether the stationary, flat banded state of $1 \mathrm{D}$ calculations will persist in $2 \mathrm{D}$, or whether it destabilises to exhibit largeamplitude interfacial fluctuations. The first step to answering this is clearly to perform a linear stability analysis of the 1D "base state" with respect to small 2D (flow, flow-gradient) perturbations corresponding to wavelike undulations along the interface.

This was first addressed within local models. McLeish [36] considered a DoiEdwards type fluid in capillary flow. He found instability to long waves, provided the high shear rate band is very narrow. As we shall see later, this is qualitatively very different from our results. (McLeish did not give the specific parameters of his calculation, so quantitative comparison is not possible.) Renardy [37] examined the stability of the local JS model in planar banded Couette flow. She found linear instability for short wavelengths (wavenumber greater than 8). For mainly historical reasons, however, she happened to confine her study to a base state corresponding to "top-jumping" $\left(T_{b}=T_{2}\right)$ and an extremely thin high-shear band. We will return below to comment on this choice in the context of our own findings.

The first observation of linear instability within the diffusive JS model was in the numerical study of [1]. This considered general band thicknesses and demonstrated, for the first time, instability with respect to long and moderatelength waves. The short-wave instability predicted in [37] was eliminated by the stabilising presence of diffusion. A subsequent non-linear numerical study showed the interface to be restabilised at the level of finite amplitude fluctuations [38].

The main contribution of the present study is a detailed analytical interpretation of the numerical findings of [1]. We start by deriving the important result that, for the limit of weak diffusion, the $2 \mathrm{D}$ linear stability properties 
of the diffusive JS model are equivalent to those of the original local model, with a "material interface" (defined below) between the bands. This equivalence was not obvious a priori, since the 1D behaviour differs so dramatically between the two models: as noted above, the diffusionless limit is singular in 1D because it has no mechanism for selecting the banding stress. The addition of weak stress diffusion thus drastically modifies the 1D global properties, by selecting a unique base state out of the continuum of possibilities.

This equivalence with the local model allows us to simplify considerably the 2D stability analysis of the diffusive case. In consequence, we are able to plot out the full spinodal boundary of instability in the phase diagram, and to predict the dispersion relations seen numerically. For a very wide range of model parameters, we find instability to waves of moderate wavelength $\lambda$ having $h^{2} \ll \lambda^{2}<L^{3} / h$ for small diffusion length, $h$ (where $L$ is a typical channel lengthscale). We also identify two possible driving terms for instability: one due to a jump across the interface in the base state shear rate, the other due to a jump in base state normal stress.

The paper is structured as follows. In section 2 we introduce our governing equations; in $\S 3$ we lay out what is known about the one-dimensional steady state solution of these equations. In $\S 4$ we set up the two-dimensional linear stability problem, and in $\S 5$ show analytically that, in the limit of small diffusion, for perturbations whose wavelength is not asymptotically short, the region between the shear bands may be considered as a material interface. This allows us to work, for the remainder of the paper, with the simpler nondiffusive model (but with a value for the selected stress in the one-dimensional base state, $T_{b}$, as selected by diffusive terms). A long-wave stability analysis is given in $\S 6$, and the results of a full numerical calculation in $\S 7$. In $\S 8$ we review our results and draw conclusions.

\section{Governing equations and dimensionless form}

The standard equations governing flow of an incompressible inertialess fluid are conservation of mass and a force balance:

$$
\nabla \cdot \underline{U}=0 \quad \nabla \cdot \underline{\underline{S}}=0
$$

in which $\underline{U}$ is the velocity field and $\underline{\underline{S}}$ is the total stress tensor. The stress consists of an isotropic pressure $P$, a Newtonian solvent term of viscosity $\eta$ plus a polymer extra stress $\underline{\underline{\underline{\Sigma}}}$

$$
\underline{\underline{S}}=-P \underline{\underline{I}}+2 \eta \underline{\underline{E}}+\underline{\underline{\Sigma}} .
$$


The extra stress evolves with dynamics based on the Johnson-Segalman equation but with two possible diffusion terms added to regularise the equations:

$$
\stackrel{\Delta}{\underline{\underline{\Sigma}}}=2 G \underline{\underline{E}}-\frac{1}{\tau} \underline{\underline{\underline{\Sigma}}}-2 \Gamma \nabla^{2} \underline{\underline{E}}+\mathcal{D} \nabla^{2} \underline{\underline{\underline{\Sigma}}}
$$

The case $\mathcal{D}=0$ reproduces Yuan's model [31]; the case $\Gamma=0$ reproduces the more usual model of Olmsted \& coworkers [29,1]; and if $\mathcal{D}=\Gamma=0$ we regain the original Johnson-Segalman model [19].

The derivative is the Johnson-Segalman form, with "slip parameter" $a$ :

$$
{\stackrel{\Delta}{\Sigma_{i j}}}^{\Delta}\left(\frac{\partial}{\partial t}+U_{k} \nabla_{k}\right) \Sigma_{i j}-\left[\Sigma_{i k} \Omega_{k j}-\Omega_{i k} \Sigma_{k j}+a\left(E_{i k} \Sigma_{k j}+\Sigma_{i k} E_{k j}\right)\right]
$$

and the tensors $\underline{\underline{E}}$ and $\underline{\underline{\Omega}}$ are based on the velocity gradient:

$$
E_{i j}=\frac{1}{2}\left(\nabla_{i} U_{j}+\nabla_{j} U_{i}\right) \quad \Omega_{i j}=\frac{1}{2}\left(\nabla_{i} U_{j}-\nabla_{j} U_{i}\right) .
$$

We scale lengths with the channel width $L$, times with the polymer relaxation time $\tau$, and stresses with the modulus $G$. The dimensionless governing equations are then:

$$
\begin{gathered}
\nabla \cdot \underline{\underline{U}}=0 \quad \nabla \cdot \underline{\underline{S}}=0 . \\
\underline{\underline{S}}=-P \underline{\underline{I}}+2 \epsilon \underline{\underline{\underline{E}}}+\underline{\underline{\underline{\Sigma}}} \\
\underline{\underline{\underline{\Sigma}}}=2 \underline{\underline{E}}-\underline{\underline{\underline{E}}}+l^{2}\left\{-2 \Delta \nabla^{2} \underline{\underline{E}}+(1-\Delta) \nabla^{2} \underline{\underline{\underline{E}}}\right\} .
\end{gathered}
$$

We have used three dimensionless parameters:

$$
\epsilon=\eta / G \tau \quad l^{2} \Delta=\Gamma G / L^{2} \quad l^{2}(1-\Delta)=\mathcal{D} \tau / L^{2} .
$$

Thus $\epsilon$ is the retardation parameter, $l$ is a characteristic diffusion lengthscale, and $\Delta$ is a selection parameter to choose between the different stress-diffusion mechanisms. The dimensional diffusion length $h$ is $l L$. Shear-banding is possible for $\epsilon<0.125$ and in all our examples we will use the value 0.05 ; it is more difficult to predict the likely physical values of other parameters, so we consider ranges $0 \leq l \leq 0.01,0.3 \leq a \leq 0.8$ and $0 \leq \Delta \leq 1$.

We will be considering a planar shear flow, bounded between two solid walls. As well as the standard velocity boundary conditions of no slip and no penetration at the walls, we require boundary conditions on the stress because of the higher derivatives introduced by the diffusion terms. We have chosen to use a boundary condition imposing no flux of extra stress at the boundaries: for a wall given by $y=$ constant this imposes the condition $\partial \underline{\underline{\Sigma}} / \partial y=\underline{\underline{0}}$ at the wall. Physically this constrains our steady flow not to have boundary layers near the walls, although (as in §3.2) boundary layer structures are permitted within the body of the fluid. 


\section{Steady-state solution}

If we impose the constraint of a steady unidirectional flow with no pressure gradient along the channel, then all flow variables depend only on the position across the channel, $y$. We denote differentiation with respect to $y$ by $D$. We also introduce new variables $T_{i j}$ based on the stress tensor $\Sigma_{i j}$ :

$$
\begin{aligned}
& T_{11}=\frac{1}{2}(1-a) \Sigma_{11}-\frac{1}{2}(1+a) \Sigma_{22} \\
& T_{22}=\frac{1}{2}(1-a) \Sigma_{11}+\frac{1}{2}(1+a) \Sigma_{22} \\
& T_{12}=\Sigma_{12} .
\end{aligned}
$$

The equations governing the flow then reduce to

$$
U_{x}=U(y) \quad U_{y}=0 \quad \dot{\gamma}=D U
$$

for the velocity field, and

$$
\begin{aligned}
& S_{11}=-P+(1-a)^{-1}\left(T_{22}+T_{11}\right) \\
& S_{12}=\epsilon \dot{\gamma}+T_{12} \\
& S_{22}=-P+(1+a)^{-1}\left(T_{22}-T_{11}\right)
\end{aligned}
$$

for the total stress. Finally, for the polymer stress we have

$$
l^{2}(1-\Delta) D^{2} T_{22}-T_{22}=0
$$

which is satisfied if $T_{22}=0$, and

$$
\begin{aligned}
\left(1-a^{2}\right) \dot{\gamma} T_{12} & =-l^{2}(1-\Delta) D^{2} T_{11}+T_{11} \\
\dot{\gamma} T_{11} & =\dot{\gamma}-T_{12}-l^{2} \Delta D^{2} \dot{\gamma}+l^{2}(1-\Delta) D^{2} T_{12} .
\end{aligned}
$$

In Couette flow, there is no pressure gradient along the channel:

$$
\frac{\partial}{\partial x} P=0
$$

and the unscaled momentum equations are

$$
T_{12}+\epsilon \dot{\gamma}=T_{b}
$$

where we recall that the shear stress $T_{b}$ is selected from the continuum of possibilities $T_{1} \leq T_{b} \leq T_{2}$ only in the diffusive model [29], and

$$
\frac{\partial P}{\partial y}=-(1+a)^{-1} D T_{11} .
$$


This last equation is the only place that the dependence of $P$ on $y$ appears, so we can solve it by setting

$$
P=-(1+a)^{-1} T_{11}
$$

Now let us look at two cases: $l$ small but non-zero (diffusive JS), and $l=0$ (non-diffusive). If $l=0$ we have the Johnson-Segalman model in its original form, with no mechanism to select the shear stress: in this (unphysical) case, shear banding can occur at any shear stress in the interval $T_{1}<T_{b}<$ $T_{2}$ (see figure 1). For any such value, there are three possible shear rates $\dot{\gamma}_{L}\left(T_{b}\right)<\dot{\gamma}_{M}\left(T_{b}\right)<\dot{\gamma}_{H}\left(T_{b}\right)$, of which a flow with $\dot{\gamma}_{M}$ would be unstable to one-dimensional perturbations. The system will therefore consist of bands of the two shear rates $\dot{\gamma}_{L}$ and $\dot{\gamma}_{H}$. There may be several of these.

If we allow $l \neq 0$, the system changes radically. The shear stress $T_{b}=T_{\text {sel }}$ is uniquely selected [39,29], and the flow will separate into shear bands of $\dot{\gamma}_{L}$ and of $\dot{\gamma}_{H}$. Between these bands, rather than a sharp interface, is a matching region of width $l$, across which the flow variables vary continuously.

In order to carry out direct comparisons between the $l=0$ and $l \neq 0$ cases, we will assume here that only one region of each shear rate is formed, and that there is then only one interface between high- and low-shear rate bands.

For definiteness, we assume that there is a band of low shear rate $\dot{\gamma}_{L}$ near the wall $y=0$, which continues up to a position $y=\kappa$. Near the wall $y=1$ (and continuing down towards $y=\kappa$ ) there is a band of high shear rate $\dot{\gamma}_{H}$. The average shear rate (and hence the speed of the upper wall) is $U_{\text {wall }}=$ $\kappa \dot{\gamma}_{L}+(1-\kappa) \dot{\gamma}_{H}$. This can equally be considered as the Weissenberg number in our nondimensionalisation. The symmetry of planar Couette flow is such that these two bands could be interchanged to produce an essentially equivalent flow: we restrict our attention to the arrangement with the low-shear band near $y=0$.

For $l \neq 0$, the two regions well away from the matching region will be denoted the outer, and the matching region of width $l$, the inner region. Each outer region is undergoing homogeneous shear flow, while the inner region can be thought of as a finite-width interface between these two phases.

\subsection{Outer solution}

Well away from the matching region, we expect derivatives of all our quantities to be at most order 1 : so if $l$ is very small we can neglect terms $l^{2} D^{2}$ and regain the equations governing one-dimensional flow of the original Johnson- 
Segalman equation. This system has been investigated thoroughly $[29,33]$. The stress components of the solution are

$$
T_{11}=\frac{\left(1-a^{2}\right) \dot{\gamma}^{2}}{1+\left(1-a^{2}\right) \dot{\gamma}^{2}} \quad T_{12}=\frac{\dot{\gamma}}{1+\left(1-a^{2}\right) \dot{\gamma}^{2}} \quad T_{22}=0
$$

and the shear stress condition $T_{12}=T_{b}-\epsilon \dot{\gamma}$ gives a cubic equation for $\dot{\gamma}$ :

$$
\left(1-a^{2}\right) \epsilon \dot{\gamma}^{3}-\left(1-a^{2}\right) T_{b} \dot{\gamma}^{2}+(1+\epsilon) \dot{\gamma}-T_{b}=0
$$

Since the neglected terms all involve derivatives of the leading-order terms, which are constants, this is an exact solution to the governing equations for each permissible value of $\dot{\gamma}$.

If $a^{2}<1$ and $\epsilon<1 / 8$, there is a range of values of the shear stress $T_{b}$ for which $T_{1}<T_{b}<T_{2}$ and equation (25) has three solutions $\dot{\gamma}_{L}, \dot{\gamma}_{M}$ and $\dot{\gamma}_{H}$, which allows the possibility of shear bands.

\subsection{Inner region}

If $l$ is small but non-zero, we do not expect a truly sharp interface between regions of high and low shear rates. Instead, in the inner region, the secondorder derivatives become $O\left(l^{-2}\right)$ and so become large enough to be important, despite being multiplied by $l^{2}$.

We take this region to be a layer centred on $\kappa$ and use a rescaling variable $y=\kappa+l \xi$. The equations become (denoting derivative with respect to $\xi$ by d):

$$
\begin{aligned}
\left(1-a^{2}\right) \dot{\gamma} T_{12} & =-(1-\Delta) d^{2} T_{11}+T_{11} \\
\dot{\gamma} T_{11} & =\dot{\gamma}-T_{12}-\Delta d^{2} \dot{\gamma}+(1-\Delta) d^{2} T_{12} \\
T_{12}+\epsilon \dot{\gamma} & =T_{b}
\end{aligned}
$$

with matching conditions at the edges of the layer for $\dot{\gamma}, T_{11}$ and $T_{12}$ in terms of the outer solution. Existence of a solution to this system which matches onto the two constant- $\dot{\gamma}$ solution branches (with $\dot{\gamma}$ dependent on $T_{b}$ ) as $\xi \rightarrow \pm \infty$ is a constraint on $T_{b}$.

This is most easily seen in the case $\Delta=1$, in which, following Lu, Olmsted \& Ball [25], (28) can be written as

$$
d^{2} \dot{\gamma}=\dot{\gamma}+\left(\epsilon \dot{\gamma}-T_{b}\right)\left(1+\left(1-a^{2}\right) \dot{\gamma}^{2}\right)
$$


Multiplying by $d \dot{\gamma}$ and integrating with respect to $\xi$ across the interface region gives a continuity condition

$$
\left[\frac{1}{2}(1+\epsilon) \dot{\gamma}^{2}+\frac{1}{4} \epsilon\left(1-a^{2}\right) \dot{\gamma}^{4}-T_{b}\left(\dot{\gamma}+\frac{1}{3}\left(1-a^{2}\right) \dot{\gamma}^{3}\right)\right]_{\dot{\gamma}_{L}}^{\dot{\gamma}_{H}}=0
$$

which, coupled with the fact that $\dot{\gamma}_{L}$ and $\dot{\gamma}_{H}$ depend on $T_{b}$, has a unique physically relevant solution

$$
T_{b}(\epsilon, a)=\frac{3}{2}\left(1-a^{2}\right)^{-1 / 2}\left(2 \epsilon-4 \epsilon^{2}\right)^{1 / 2} .
$$

A similar result (with a different function $T_{b}(\epsilon, a)$ ) is true for other values of $\Delta$. For reference, we note that at $\epsilon=0.05$, the extremal selected values are

$$
\Delta=0: \quad T_{b}=0.48284\left(1-a^{2}\right)^{-1 / 2} ; \quad \Delta=1: T_{b}=0.45\left(1-a^{2}\right)^{-1 / 2} .
$$

In this way, the addition of weak diffusion is seen to regularise the equations by selecting one possible value $T_{b}$ out of the continuum of possibilities $T_{1}<$ $T_{b}<T_{2}$ at which a one-dimensional shear-banded solution can occur. This one-dimensional solution will be taken as the relevant base state about which we analyse two-dimensional perturbations (in the flow, flow gradient plane) in the remainder of the paper.

\section{Linearised equations}

We now add a small perturbation proportional to $\mathcal{E}$, such that

$$
\begin{gathered}
\underline{U}=(U+u \mathcal{E}, v \mathcal{E}) \quad P=P_{\text {base }}+p \mathcal{E} \\
\underline{\underline{S}}=\left(\begin{array}{c}
S_{11}+s_{11} \mathcal{E} S_{12}+s_{12} \mathcal{E} \\
S_{12}+s_{12} \mathcal{E} S_{22}+s_{22} \mathcal{E}
\end{array}\right) \quad \underline{\underline{T}}=\left(\begin{array}{cc}
T_{11}+t_{11} \mathcal{E} & T_{12}+t_{12} \mathcal{E} \\
T_{12}+t_{12} \mathcal{E} & t_{22} \mathcal{E}
\end{array}\right)
\end{gathered}
$$

in which

$$
\mathcal{E}=\delta \exp (i k x-i \omega t)
$$

for a small parameter $\delta$, and we will use $D$ to denote differentiation with respect to $y$. We have introduced the dimensionless wavenumber $k=2 \pi L / \lambda$ for waves of wavelength $\lambda$. We can now linearise the equations about the base state in this small parameter, to obtain our new governing equations for evolution of the linear system. For a fixed real wavenumber $k$, if we obtain a valid solution with $\operatorname{Im}(\omega)>0$ then the system is linearly unstable. This analysis is necessarily restricted to considering instabilities which manifest at linear order; any instability (like the high-Reynolds number instability of 
laminar flows) which appears only at nonlinear order will not be captured here. The mass conservation equation is

$$
i k u+D v=0
$$

This is automatically satisfied by the introduction of a streamfunction $\psi$ with $v=-i k \psi$ and $u=D \psi$, such that the perturbation velocity $\underline{u} \mathcal{E}=\underline{\nabla} \times(\psi \underline{\hat{z}} \mathcal{E})$. The remaining governing equations for the force balance become

$$
\begin{aligned}
& \qquad i k s_{11}+D s_{12}=0 \quad i k s_{12}+D s_{22}=0 \\
& s_{11}=-p+2 \epsilon i k D \psi+(1-a)^{-1}\left(t_{22}+t_{11}\right) \\
& s_{12}=\epsilon\left(D^{2} \psi+k^{2} \psi\right)+t_{12} \\
& s_{22}=-p-2 \epsilon i k D \psi+(1+a)^{-1}\left(t_{22}-t_{11}\right)
\end{aligned}
$$

and for the polymer stress we have:

$$
\begin{gathered}
\left(-i \omega+i k U+1+l^{2} k^{2}\right) t_{22}=l^{2}(1-\Delta) D^{2} t_{22}+2 i k a l^{2} \Delta\left(D^{2}-k^{2}\right) D \psi \\
+2 k^{2} a \psi T_{12}+2 i k a\left(T_{11}-1\right) D \psi \\
\left(-i \omega+i k U+1+l^{2} k^{2}\right) t_{11}=l^{2}(1-\Delta) D^{2} t_{11}-2 i k l^{2} \Delta\left(D^{2}-k^{2}\right) D \psi \\
+\left(1-a^{2}\right) \dot{\gamma} t_{12}+i k \psi D T_{11}+2 i k D \psi+T_{12}\left[\left(1-a^{2}\right) D^{2} \psi-k^{2}\left(1+a^{2}\right) \psi\right] \\
\left(-i \omega+i k U+1+l^{2} k^{2}\right) t_{12}=l^{2}(1-\Delta) D^{2} t_{12}-l^{2} \Delta\left(D^{4}-k^{4}\right) \psi \\
-\dot{\gamma} t_{11}+i k \psi D T_{12}+\left(1-T_{11}\right)\left(D^{2}+k^{2}\right) \psi+2\left(1-a^{2}\right)^{-1} k^{2} \psi T_{11} .
\end{gathered}
$$

\section{Equivalence of the stability analyses at $l \rightarrow 0$ and $l=0$}

\subsection{Introduction}

The limit $l \rightarrow 0$ is a singular limit, in the sense that the coefficient of the highest derivative in the governing equations is multiplied by $l^{2}$. As discussed above, therefore, the one-dimensional solutions to equations (10-19) are very different for small $l$ from $l=0$ : for $l \neq 0$ the shear stress $T_{b}$ at which a banded state can exist is uniquely selected, whereas for $l=0$ banding can occur at any shear stress $T_{1}<T_{b}<T_{2}$.

In this section we consider the limit of very small $l$ in the two-dimensional stability problem, and show that, in contrast to the one-dimensional case, 
the limit $l \rightarrow 0$ is equivalent to $l=0$ (with suitable interfacial boundary conditions), for any given base state. To do this, we start by considering the full diffusive model, in the asymptotic limit of small $l$. As $l \rightarrow 0$ the diffusion terms become unimportant everywhere in the flow apart from in the inner region, which has dimension $l$. This region separates two phases of flow at different shear rates, in which the governing equations are those of the original (non-diffusive) Johnson-Segalman equation.

The crucial question to address here is what boundary conditions are imposed at the edge of each of these phases as a result of the structure of the inner solution. Our central finding is that these conditions are the same in the limit of a thin interface as they would be for the case of an infinitesimally sharp material interface (that is, an interface across which material does not pass) with $l=0$. Accordingly, we now summarise the case of a sharp (diffusion-free) material interface $l=0$ before proceeding with our analysis of weak diffusion, $l \rightarrow 0$.

McLeish [36] has argued that if the functional relating current stress to strain history is well-behaved, a small perturbation to the flow can only cause a small perturbation to the stress in any fluid element. Thus material can never be transported across a sharp interface since this would cause an order 1 change in the stress for that material.

This physical argument leads to a kinematic boundary condition: if the interface is defined by points $y=\bar{\eta}+\zeta(x, \bar{\eta}, t)$ (where $\bar{\eta}$ is the Lagrangian cross-channel coordinate) then

$$
\frac{D \bar{\eta}}{D t}=\frac{\partial \bar{\eta}}{\partial t}+\underline{u} \cdot \underline{\nabla} \bar{\eta}=0
$$

This boundary condition is standard for an interface separating two different materials: in particular, Renardy [37] applied it to two phases of a shearbanded Johnson-Segalman fluid. For our linear perturbation problem, if the interface $\bar{\eta}=\kappa$ is displaced from $y=\kappa$ to $y=\bar{\eta}+\zeta \mathcal{E}$, then since $\bar{\eta}=y-\zeta \mathcal{E}$, the kinematic boundary condition (correct to linear order) is expressed as

$$
(-i \omega+i k U(\kappa)) \zeta=-i k \psi(\kappa)
$$

Then imposing continuity of velocity and traction across the interface gives boundary conditions for the jumps in $\psi, D \psi$ and $s_{i j}$ across the interface.

With this summary of the diffusion-free sharp material interface in mind, we now proceed to show that the case $l \rightarrow 0$ is equivalent to it. 


\subsection{Asymptotic analysis}

We now consider the asymptotic limit $l \rightarrow 0$. We scale our perturbation flow so that the values of standard quantities $\left(e . g . \psi, D \psi, t_{12}\right)$ are order 1 in the outer regions. Within the inner region we pose the following asymptotic series (in which superscripts on $\psi, s_{i j}, t_{i j}$ and $p$ are for labelling only, while those on $l$ indicate powers):

$$
\begin{aligned}
\psi & \sim \psi^{0}+l \psi^{1}+l^{2} \psi^{2}+\cdots & t_{i j} & \sim l^{-1} t_{i j}^{-1}+t_{i j}^{0}+\cdots \\
p & \sim l^{-1} p^{-1}+p^{0}+\cdots & s_{i j} & \sim l^{-1} s_{i j}^{-1}+s_{i j}^{0}+\cdots
\end{aligned}
$$

These scalings are not obvious a priori; rather, the existence of a solution with these scalings will justify its choice. As before, we scale lengths within the interfacial region as $\xi=(y-\kappa) / l$, giving $l D=d$. This analysis will be valid as long as this lengthscale is well separated from other lengthscales in the flow: in particular, the arguments and analysis below will not apply for very short waves for which $k \sim l^{-1}$ : we restrict our analysis to the case $k l \ll 1$.

We also pose a series for the eigenvalue $\omega$ :

$$
\omega \sim \omega_{0}+l \omega_{1}+\cdots
$$

Well away from the interface, all quantities are at most order 1, which we have ensured by scaling the whole perturbation. This gives a series of conditions on our variables as $\xi \rightarrow \pm \infty$. In particular, since $D \psi, t_{i j}$ and $s_{i j}$ must be finite, with no contribution at $O\left(l^{-1}\right)$, we have the conditions

$$
d \psi^{0} \rightarrow 0, t_{i j}^{-1} \rightarrow 0 \text { and } s_{i j}^{-1} \rightarrow 0 \text { as } \xi \rightarrow \pm \infty .
$$

We split the governing equations into successive orders of $l$. At order $l^{-2}$ we have, from the stress equations, simply (from (39))

$$
d^{2} \psi^{0}=0
$$

which, along with the matching condition (49) gives $\psi^{0}=$ constant $=\alpha_{0}$. This means that the streamfunction $\psi$ (or velocity in the $y$-direction) is continuous across the interface region, and $\alpha_{0}$ is the value of $\psi$ at the interface.

At $O\left(l^{-2}\right)$ in the momentum equations, we have:

$$
d s_{12}^{-1}=0 \quad d s_{22}^{-1}=0
$$

which, along with (49), give $s_{12}^{-1}=s_{22}^{-1}=0$. Then the stress equations (38)(40) at $O\left(l^{-1}\right)$ give

$$
p^{-1}=-(1+a)^{-1} t_{11}^{-1}, \quad s_{11}^{-1}=2\left(1-a^{2}\right)^{-1} t_{11}^{-1},
$$




$$
\epsilon d^{2} \psi^{1}+t_{12}^{-1}=0
$$

The equations governing $t_{i j}^{-1}$ at order $O\left(l^{-1}\right)$ become:

$$
\begin{gathered}
\left(-i \omega_{0}+i k U(\kappa)+1\right) t_{11}^{-1}=(1-\Delta) d^{2} t_{11}^{-1}+\left(1-a^{2}\right) \dot{\gamma} t_{12}^{-1} \\
+i k \alpha_{0} d T_{11}+\left(1-a^{2}\right) T_{12} d^{2} \psi^{1} \\
\left(-i \omega_{0}+i k U(\kappa)+1\right) t_{12}^{-1}=(1-\Delta) d^{2} t_{12}^{-1}-\Delta d^{4} \psi^{1}-\dot{\gamma} t_{11}^{-1} \\
+i k \alpha_{0} d T_{12}+\left(1-T_{11}\right) d^{2} \psi^{1} \\
t_{22}^{-1}=0 .
\end{gathered}
$$

We now introduce a quantity

$$
\zeta=-i k \alpha_{0} /\left(-i \omega_{0}+i k U(\kappa)\right)
$$

Equations (53)-(55) then give the following system:

$$
\begin{gathered}
\epsilon d^{2} \psi^{1}+t_{12}^{-1}=0 \\
t_{11}^{-1}=(1-\Delta) d^{2} t_{11}^{-1}+\left(1-a^{2}\right) \dot{\gamma} t_{12}^{-1}+\left(1-a^{2}\right) T_{12} d^{2} \psi^{1} \\
-\left(-i \omega_{0}+i k U(\kappa)\right)\left[t_{11}^{-1}+\zeta d T_{11}\right] \\
t_{12}^{-1}=(1-\Delta) d^{2} t_{12}^{-1}-\Delta d^{4} \psi^{1}-\dot{\gamma} t_{11}^{-1}+\left(1-T_{11}\right) d^{2} \psi^{1} \\
-\left(-i \omega_{0}+i k U(\kappa)\right)\left[t_{12}^{-1}+\zeta d T_{12}\right]
\end{gathered}
$$

which is solved by

$$
d^{2} \psi^{1}=-\zeta d \dot{\gamma}, \quad t_{12}^{-1}=-\zeta d T_{12}, \quad t_{11}^{-1}=-\zeta d T_{11},
$$

all of which tend to zero for large $|\xi|$ as required. These are the first derivatives (in the flow-gradient direction) of the corresponding base-state quantities, which for long waves are the perturbations we would expect from a simple displacement of the interface by an amount $\zeta$. Integrating $d^{2} \psi^{1}$ gives

$$
d \psi^{1}=\alpha_{1}-\zeta \dot{\gamma}
$$

We note that (52) also gives

$$
s_{11}^{-1}=2\left(1-a^{2}\right)^{-1} t_{11}^{-1}=-2\left(1-a^{2}\right)^{-1} \zeta d T_{11}=-\zeta d S_{11} .
$$

At $O\left(l^{-1}\right)$, the two momentum equations give

$$
i k s_{11}^{-1}+d s_{12}^{0}=0 \quad i k s_{12}^{-1}+d s_{22}^{0}=0
$$

and thus

$$
s_{12}^{0}=\alpha_{2}+i k \zeta S_{11} \quad s_{22}^{0}=\alpha_{3} .
$$




\subsection{Physical meaning}

We have now calculated all the singular components of the perturbation flow for the asymptotic limit $l \rightarrow 0$. What conclusions can we draw about the behaviour of the outer quantities (which are not directly affected by diffusion) at the edges of the inner layer? Looking at velocities and stresses (and recalling that $D \psi \sim l^{-1} d \psi$ so that the order 1 term in $D \psi$ comes from $\psi^{1}$ ), we have the following conditions (to within corrections of $O(l)$ ):

$$
\psi\left(\kappa_{-}\right)=\psi\left(\kappa_{+}\right)=\alpha_{0}
$$

which follows from (50),

$$
D \psi\left(\kappa_{-}\right)+\zeta \dot{\gamma}\left(\kappa_{-}\right)=D \psi\left(\kappa_{+}\right)+\zeta \dot{\gamma}\left(\kappa_{+}\right)=\alpha_{1}
$$

from (61), and two conditions from (65):

$$
\begin{gathered}
s_{12}\left(\kappa_{-}\right)-i k \zeta S_{11}\left(\kappa_{-}\right)=s_{12}\left(\kappa_{+}\right)-i k \zeta S_{11}\left(\kappa_{+}\right)=\alpha_{2} \\
s_{22}\left(\kappa_{-}\right)=s_{22}\left(\kappa_{+}\right)=\alpha_{3}
\end{gathered}
$$

along with the definition

$$
\zeta=-i k \alpha_{0} /\left(-i \omega_{0}+i k U(\kappa)\right) \quad\left(-i \omega_{0}+i k U(\kappa)\right) \zeta=-i k \psi(\kappa) .
$$

We have used the notation $X\left(\kappa_{-}\right)$to denote the value of variable $X$ in the limit $y \rightarrow \kappa$ of the outer region given by $0 \leq y<\kappa$; similarly, $X\left(\kappa_{+}\right)$denotes the value as $y \rightarrow \kappa$ in the outer region $\kappa<y \leq 1$.

These equations exactly reproduce those for a sharp material interface with displacement $\zeta$ from its original position and a discontinuity in both $\dot{\gamma}$ and $S_{11}$ across it in the base state. If we identify the quantity $\zeta$ as a displacement in the interface, then the conditions (66-67) correspond to continuity of the total fluid velocity at the interface, and (68-69) come from the momentum equations, and correspond to continuity of the total traction $(\underline{\underline{\Sigma}}+\underline{\underline{\sigma}} \mathcal{E}) \cdot \underline{n}$ at the interface, where $\underline{n}$ is the normal to the displaced interface. Of course the interface itself has width of order $l$, so only in the asymptotic limit $l \rightarrow 0$ do we truly have an interface to consider, which is why the analysis above is only valid in this limit. Note that the original Johnson-Segalman equations governing a linear perturbation are fourth-order in $\psi$, and equations (66-67) supply four boundary conditions at the interface, as we require.

In this limit $l=0$ but with a finite interface displacement, we have effectively exceeded the validity of our original linearisation. When we linearised about the base state, we implicitly assumed that the scale of all perturbation velocities, displacements and so on was much smaller than any other quantity in the problem, and in particular that displacements must be smaller than 
the width of the interface region, $l$. In taking the asymptotic limit $l \rightarrow 0$ we have continued the assumption that displacements are small relative to the interface size: so this asymptotic limit is not founded on a well-posed physical problem. This makes it even more surprising that the limit should correspond exactly to the physical problem with an infinitely sharp material interface, in which the (small) displacement is much larger than the (zero) interface width.

If we consider a perturbation to consist of an initial perturbation of the interface (this assumption is not always valid, as demonstrated recently by Kupferman [40]), then the driving terms for the perturbation are the $\zeta$ terms in (67) and (68), which depend on discontinuities in the base-state. If the shear rate were continuous across the interface, the only driving term would be the discontinuity in $S_{11}$ in (68); this would then allow us to recapture the normal stress instability mechanism elucidated by Hinch et al. [2]. The discontinuity in shear rate in (67) provides another, more complex, mechanism for instability, which is not yet fully understood.

At order $l$, however, equations (66-69) will not be satisfied so the diffusion does alter the zero-transport property. This is to be expected if the stress diffusion derives physically from diffusive transport of individual polymer molecules carrying their stress history with them: we would expect material to travel across the interface, a distance of $l$, with diffusive velocity of $l^{2}$, on a timescale of order $l^{-1}$. This timescale corresponds to a contribution of order $l$ to $\omega$, i.e. the next term in the asymptotic series, $\omega_{1}$.

\subsection{Wall boundary conditions}

In our discussion of the boundary conditions applying across the diffusive interface layer, we have implicitly assumed that this layer is the only place in the perturbation flow where diffusion is important. However, the stress boundary conditions $D \sigma_{i j}=0$ at the walls are not automatically satisfied by the perturbation flow of a pure Johnson-Segalman fluid. We would expect that a stress boundary layer would form at each wall, matching between our outer solution and the no-flux boundary conditions.

This does indeed occur, but the influence of these stress boundary layer regions does not extend into the bulk of the flow, and they do not affect the stability of the flow. They are standard boundary layers as one would expect for what is essentially an advection-diffusion equation in extra stress. Although we are not presenting an analytical justification of the assertion that their effect is negligible, the close match between our results (neglecting the wall boundary layers) and those of [1] and of $\S 7.2$ at small finite $l$ with no such assumptions, justifies our claim. 


\section{Long wave analysis $k \ll 1$ at $l=0$}

We showed in Section 5 that as long as the wavenumber $k$ is not very large (we assumed in Section 5.2 that $k l \ll 1$ ), the interface between the two phases of the flow may be treated as a material interface, with corrections appearing at $O(l)$ for small $l$. A typical estimate of $l$ (the ratio of mesh size to channel width in [41]) is $2 \times 10^{-4}$, so the waves which are excluded from our analysis are very short indeed.

In summary, for small diffusion, the diffusive terms in the outer region do not affect the flow at any physically important level, so the only rôle played by diffusion is in the "boundary" conditions that it imposes on the outer at the edges of the inner, interface, region. We have just shown that these are equivalent to treating the interface as a material interface in the case $l=0$ : so the solution to the $l=0$ problem with a material interface must be the same as the $l \rightarrow 0$ limit of the diffusive problem. This equivalence is verified in Section 7 by the agreement between the $l=0$ results of this paper with earlier numerical results by one of the authors [1] for small $l$.

This finding allows us now to consider the simpler (although artificial) limit $l=0$, in which the diffusive terms play no part at all, and the base state quantities $\dot{\gamma}, T_{11}$ and $T_{12}$ are constant within each fluid phase, and treat the interface as a material surface. We are now dealing with the original JohnsonSegalman fluid [19] and considering a stability problem which was studied by Renardy [37] in 1995, although we have some analytical results for long waves where her work was purely numerical. However, as discussed above, for planar Couette flow of a Johnson-Segalman fluid in the non-monotonic region of the flow curve, there is no mechanism for selection of the shear stress. Any shear stress that intersects both the low- and high-shear-rate parts of the flow curve, $T_{b}$ with $T_{1}<T_{b}<T_{2}$ (figure 1 ) is a possible solution to the governing equations, with the choice of shear stress (for a given wall velocity) governing the position of the interface. Renardy's solution to this difficulty was to assume top-jumping, that is, the maximum value of the shear stress for which the flow curve is multi-valued. We, however, are using this local model as an approximation to the non-local case $l \neq 0$, so we adopt the unique value of $T_{b}$ selected by use of the gradient terms in the stress-diffusive non-local case.

In the case of long waves, $k \rightarrow 0, p, s_{11}$ and $s_{22}$ are larger than the other perturbation quantities by a factor of $k^{-1}$, and the eigenvalue $\omega$ is expected to be of order $k$, based on prior experience of long-wave interfacial instabilities [2]. If we scale these quantities accordingly: $p=k^{-1} \bar{p}, s_{i i}=k^{-1} \bar{s}_{i i}, \omega=k \bar{\omega}$, then the governing equations within each fluid, from (37-43), become, for the force 
balance:

$$
i \bar{s}_{11}+D s_{12}=0 \quad i k^{2} s_{12}+D \bar{s}_{22}=0,
$$

for the total stress:

$$
\begin{aligned}
& \bar{s}_{11}=-\bar{p}+2 \epsilon i k^{2} D \psi+(1-a)^{-1} k\left(t_{22}+t_{11}\right) \\
& s_{12}=\epsilon\left(D^{2}+k^{2}\right) \psi+t_{12} \\
& \bar{s}_{22}=-\bar{p}-2 \epsilon i k^{2} D \psi+(1+a)^{-1} k\left(t_{22}-t_{11}\right),
\end{aligned}
$$

and for the polymer extra stress:

$$
\begin{gathered}
(-i k \bar{\omega}+i k U+1) t_{22}=2 a i k\left(T_{11}-1\right) D \psi+2 a k^{2} \psi T_{12} \\
(-i k \bar{\omega}+i k U+1) t_{11}=2 i k D \psi+\left(1-a^{2}\right) \dot{\gamma} t_{12} \\
+T_{12}\left(\left(1-a^{2}\right) D^{2} \psi-\left(1+a^{2}\right) k^{2} \psi\right) \\
(-i k \bar{\omega}+i k U+1) t_{12}=\left(1-T_{11}\right)\left(D^{2}+k^{2}\right) \psi-\dot{\gamma} t_{11}+2\left(1-a^{2}\right)^{-1} T_{11} k^{2} \psi
\end{gathered}
$$

If the interface has been displaced from its original position $\bar{\eta}=\kappa$ to a new position $\bar{\eta}=\kappa+\zeta \exp (i k x-i \omega t)$, then the conditions of continuity of velocity and traction across the interface give us continuity of the following quantities at $y=\kappa$ :

$$
-i k \psi \quad \dot{\gamma} \zeta+D \psi \quad-i k \zeta S_{11}+s_{12} \quad \bar{s}_{22}
$$

in agreement with the results of (66-69) for $l \rightarrow 0$.

Finally, the condition that the interface should be a material surface, equivalent to (70), gives

$$
(-\bar{\omega}+U(\kappa)) \zeta=-\psi(\kappa) .
$$

This system of equations is essentially fourth-order in $\psi$, so the general solution within one shear band will contain four unknowns. The no-slip, no-penetration boundary conditions on the walls remove two unknowns in each band, leaving a total of four unknowns to be determined through these jump conditions at the interface. Since there are four jump conditions here, and no driving term not proportional to $\psi$, the existence of a non-zero solution to the problem is the eigenvalue condition which allows $\omega$ to be fixed.

We expand each perturbation quantity as a regular power series in $k$. A critical quantity in each fluid phase is the marginal viscosity, that is, the slope of the stress-shear-rate relation $\sigma(\dot{\gamma})$ at our selected shear rate:

$$
\bar{\mu}=\frac{\mathrm{d} S_{12}}{\mathrm{~d} \dot{\gamma}}=\frac{\mathrm{d}}{\mathrm{d} \dot{\gamma}}\left(\epsilon \dot{\gamma}+T_{12}\right)=\epsilon+\frac{\left(1-2 T_{11}\right)}{\left(1+\left(1-a^{2}\right) \dot{\gamma}^{2}\right)} .
$$

Using $X_{L}$ to denote the value of quantity $X$ in the lower shear-rate phase, and $X_{H}$ to denote its value in the higher shear-rate phase, the solution at leading 
order in $k$ is

$$
s_{12}=\bar{\mu}_{L} D^{2} \psi_{L}=\bar{\mu}_{H} D^{2} \psi_{H}=A y+B \quad s_{11}=s_{22}=-p=i A
$$

where $A$ and $B$ are constants. The physical interpretation of this result is that, to this order, the perturbation consists of simply displacing the interface: a one-dimensional perturbation. If $\omega=0$ (as it is to this order in $k$ ), then the system is still on the "adiabatic" constitutive curve of figure 1, but with a modified stress $T_{b}$. Thus the change in stress is

$$
s_{12}=\Delta S_{12}=\frac{\mathrm{d} S_{12}}{\mathrm{~d} \dot{\gamma}} \Delta \dot{\gamma}=\bar{\mu} D^{2} \psi
$$

since the change in shear rate at this order is $D^{2} \psi$.

Integration of $D^{2} \psi$ subject to zero perturbation velocity on the channel walls determines $\psi$ in each phase, and the interfacial continuity conditions then give the values of the two constants:

$$
\begin{aligned}
A & =\frac{6 \zeta\left[\bar{\mu}_{H} \kappa^{2}-\bar{\mu}_{L}(\kappa-1)^{2}\right] \bar{\mu}_{L} \bar{\mu}_{H}\left(\dot{\gamma}_{H}-\dot{\gamma}_{L}\right)}{\left[\bar{\mu}_{H} \kappa^{2}-\bar{\mu}_{L}(\kappa-1)^{2}\right]^{2}-4 \bar{\mu}_{L} \bar{\mu}_{H} \kappa(\kappa-1)}, \\
B & =\frac{2 \zeta\left[\bar{\mu}_{L}\left(\kappa^{3}-3 \kappa+2\right)-\bar{\mu}_{H} \kappa^{3}\right] \bar{\mu}_{L} \bar{\mu}_{H}\left(\dot{\gamma}_{H}-\dot{\gamma}_{L}\right)}{\left[\bar{\mu}_{H} \kappa^{2}-\bar{\mu}_{L}(\kappa-1)^{2}\right]^{2}-4 \bar{\mu}_{L} \bar{\mu}_{H} \kappa(\kappa-1)} .
\end{aligned}
$$

The eigenvalue at this order is real, which corresponds to the perturbation travelling in the flow direction, and is given by

$$
\omega \sim \dot{\gamma}_{L} \kappa k+\frac{2 \bar{\mu}_{L} \bar{\mu}_{H} \kappa^{2}(\kappa-1)^{2}\left(\dot{\gamma}_{H}-\dot{\gamma}_{L}\right) k}{\left[\bar{\mu}_{H} \kappa^{2}-\bar{\mu}_{L}(\kappa-1)^{2}\right]^{2}-4 \bar{\mu}_{L} \bar{\mu}_{H} \kappa(\kappa-1)}+O\left(k^{2}\right) .
$$

The imaginary part of $\omega$ determines the stability or instability of the flow $(\operatorname{Im}(\omega)<0$ for stable flow, $\operatorname{Im}(\omega)>0$ for unstable flow). This appears at the next order. This problem can still be solved analytically, but the result is too unwieldy to reproduce here.

Instead, in figure 2 we show the stability boundaries for this long-wave mode of perturbation, plotted in the parameter plane of $T_{b} \sqrt{\left(1-a^{2}\right)}$ against $\kappa$.

It will be seen that the range of parameter values over which the flow is unstable includes most of the available values of $\kappa$, with the exception of values giving an interface very close to one of the walls. In particular, for the shear stress which is selected by the stress-diffusion model $\Delta=0$, there is instability for $0.108<\kappa<0.933$.

In the next section we give the results of numerical calculations at finite values of $k$, and we will show these analytical asymptotes along with those numerical results. 


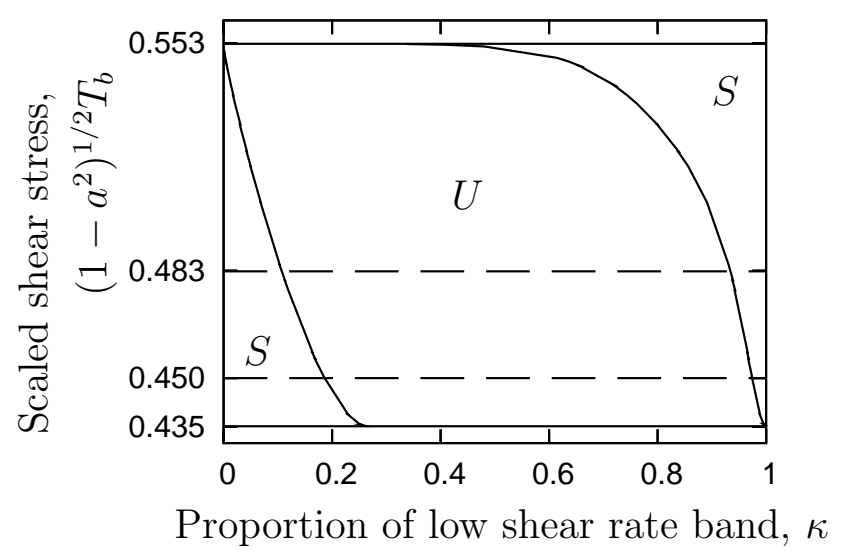

Fig. 2. Stability boundaries in parameter space for long-wave perturbations (stability or instability at order $k^{2}$ ). The $x$-axis is $\kappa$, the proportion of low-shear-rate fluid in the base flow; the $y$-axis is the scaled shear stress $\left(1-a^{2}\right)^{1 / 2} T_{b}$. The curves shown are for $\epsilon=0.05$, and these boundaries are independent of the value of $a$. The solid horizontal lines show the minimum and maximum possible values of $\left(1-a^{2}\right)^{1 / 2} T_{b}$, being 0.435207 and 0.552806 respectively for this value of $\epsilon$. The dotted horizontal lines show the selected values of $\left(1-a^{2}\right)^{1 / 2} T_{b}$ for the two limiting diffusive models: Olmsted's model $(\Delta=0)$ predicts $\left(1-a^{2}\right)^{1 / 2} T_{b}=0.48284$, while Yuan's model $(\Delta=1)$ has $\left(1-a^{2}\right)^{1 / 2} T_{b}=0.45$ for $\epsilon=0.05$. $U$ denotes the unstable region (typically for two roughly equal shear bands) and $S$ the stable regions (typically one or other shear band being very narrow). The stable regions here have only been shown to be stable to asymptotically long waves: as we see in section 7 , most of these parameters do show instability for some value of the wavenumber, $k$.

\section{$7 \quad$ Numerical Results}

In order to access perturbations with moderate or short wavelengths, we solve the linearised equations numerically. The scheme used is a shooting method, in which a value of $\omega$ is guessed and the Newton-Raphson method is used to find the true value. The equations are integrated inwards from each wall and the jump conditions at the interface provide the dispersion relation through a determinant condition as introduced by Ho \& Denn [42].

\subsection{Most unstable mode}

In this section we choose as illustrative the parameters $\epsilon=0.05$ and $a=$ 0.3 , and investigate the wavenumber-dependence of the instability. We use the nonlocal model with stress diffusion (i.e. $\Delta=0$ ) to select the shear stress $T_{b}=0.506158$ of the one-dimensional base state, but the subsequent two-dimensional linear stability calculations are carried out using the local Johnson-Segalman model. 


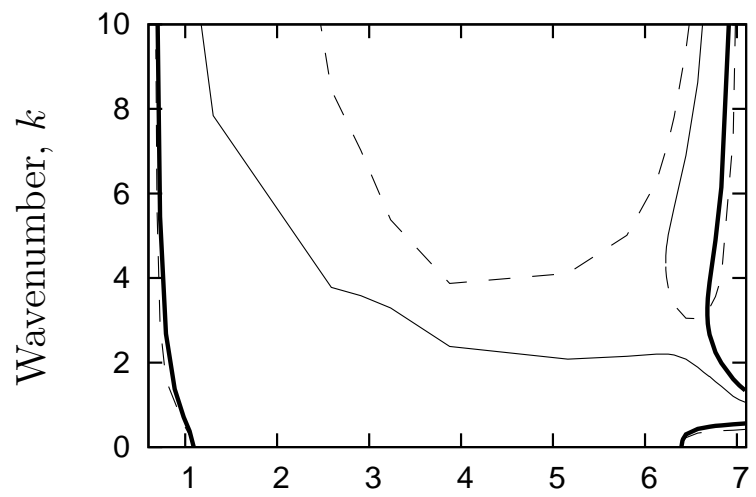

Average shear rate, $U_{\text {wall }}$

Fig. 3. The dependence of the growth rate of instability on the average shear rate $U_{\text {wall }}$ and on wavenumber, $k$. The parameters are $\epsilon=0.05, a=0.3$ and the shear stress is that selected by the non-local model with stress diffusion, $T_{b}=0.506158$. The bold solid curves mark the boundaries between instability and stability (the unstable region being the centre of the plot), the thin solid lines mark local maxima in the plot of growth rate against wavenumber, and the dashed curves mark local minima in the same plot.

We vary the average shear rate across the channel, $U_{\text {wall }}$, which changes the proportion of the lower shear rate band. Our nondimensionalisation means that $U_{\text {wall }}$ is the same as the Weissenberg number. The behaviour of the instability against wavenumber of the perturbation changes as $U_{\text {wall }}$ varies. It is only for extremely low shear-rates, that is, thin high-shear bands that this flow is stable for all wavenumbers. In figure 3 we plot various curves in the $U_{\text {wall }}-k$ plane. The thick solid curves mark the borderline between unstable and stable parameters. For most values of $U_{\text {wall }}$ in the shear-banding region $0.661<U_{\text {wall }}<7.089$ these curves do not appear as the perturbations at all wavenumbers are unstable.

The thin solid curves gives the wavenumbers at which the growth rate reaches a local peak. Where there is only one such curve (for example, at $U_{\text {wall }}=4$ ), it is tempting to regard this as the most unstable mode; however, within the confines of our local approximation to the diffusive Johnson-Segalman fluid, there is an instability to very short waves [37], with a growth rate independent of $U_{\text {wall }}$, and for many layer arrangements this is in fact the most unstable mode. For our illustrative parameters, the growth rate of the $k \rightarrow \infty$ mode is 0.533 , and this mode is the most unstable for $U_{\text {wall }}>3.2$. For lower values of $U_{\text {wall }}$ the most unstable mode is the peak at the lowest wavenumber, although this wavenumber also becomes very large as $U_{\text {wall }} \rightarrow \dot{\gamma}_{L}$. In practice, as we shall see in $\S 7.2$, for realistic non-zero values of $l$ we expect diffusive effects to damp very short-wave instabilities, and the peak growth rate will always occur at finite $k$ : in $\S 7.2 .1$ we will show how the maximum growth rate varies with average shear rate, with diffusion added in a semi-empirical way. 


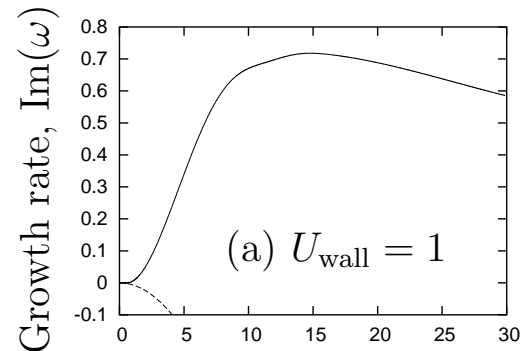

Wavenumber, $k$

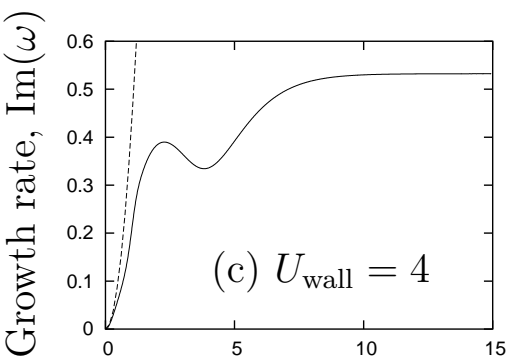

Wavenumber, $k$

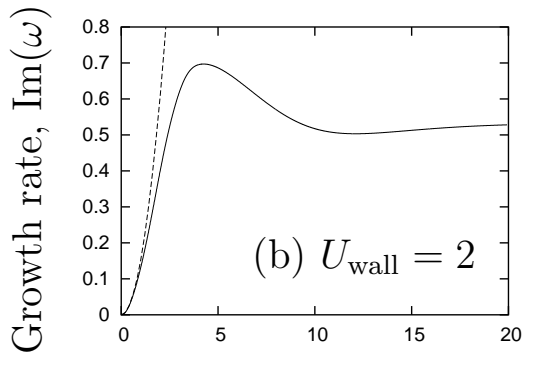

Wavenumber, $k$

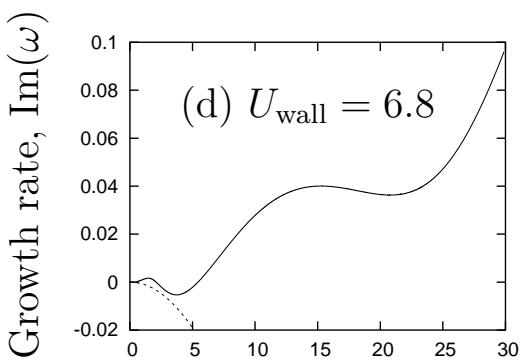

Wavenumber, $k$

Fig. 4. Plots of growth rate against wavenumber for four different values of $U_{\text {wall }}$, the average shear rate. The parameters here are $\epsilon=0.05, a=0.3, l=0$, and $T_{b}=0.506158$, the value predicted by the stress-diffusion model. In each case the order $k^{2}$ behaviour of long waves is a dotted curve. (a) $U_{\text {wall }}=1$. Long waves are stable with decay rate $\operatorname{Im}(\omega) \sim-0.0060 k^{2}$ and instability begins at $k>0.65$ (not discernible on the scale of the plot). The region $k>30$, in which the growth rate tends smoothly to 0.533 from above, is omitted to allow a clearer view of the behaviour for longer waves. (b) $U_{\text {wall }}=2$. Long waves are unstable with growth rate $\operatorname{Im}(\omega) \sim 0.15 k^{2}$, and the most unstable mode is at $k=4.3$ with growth rate 0.698 . (c) $U_{\text {wall }}=4$. Long waves are unstable with growth $\operatorname{rate} \operatorname{Im}(\omega) \sim 0.42 k^{2}$, and there is a peak in growth rate at $k=2.3$, but the most unstable mode is $k \rightarrow \infty$. (d) $U_{\text {wall }}=6.8$. Here, as in (a), asymptotically long waves are stable. Long waves have decay rate $\operatorname{Im}(\omega) \sim-0.00076 k^{2}$. We have omitted the region $k>30$, in which the growth rate tends smoothly to 0.533 from below.

In figure 4 we give four plots of growth rate against wavenumber, to illustrate the different types of behaviour seen in figure 3. The parameters chosen are $U_{\text {wall }}=1$, for which long waves are stable, and instability arises at finite $k ; U_{\text {wall }}=2$, which has one maximum which is the most unstable mode; $U_{\text {wall }}=4$, which has a single local maximum but for which the most unstable mode is for asymptotically short waves; and $U_{\text {wall }}=6.8$, for which (again) long waves are stable, and two separate unstable regions $0.51<k<2.1$ and $k>5.45$ are observed. In each case the behaviour as $k \rightarrow \infty$ is the short-wave instability predicted by Renardy [37], with growth rate 0.533 , and the longwave behaviour matches the analytic calculation of section 6 . The long-wave asymptotes are plotted along with the numerical calculations. 

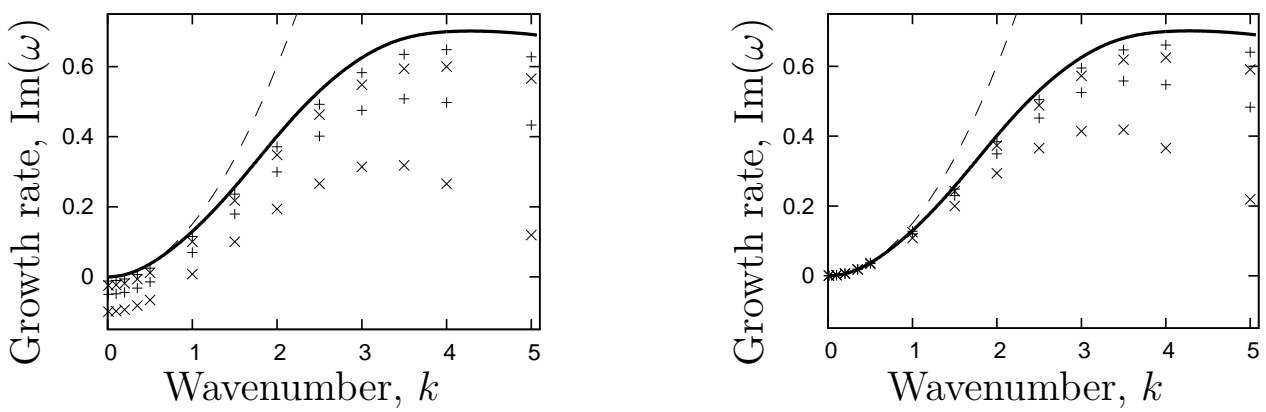

Fig. 5. Growth rate $\operatorname{Im}(\omega)$ plotted against wavenumber, $k$ for Couette flow at $\epsilon=0.05, a=0.3, U_{\text {wall }}=2, T_{b}=0.506158$. Curves: $l=0$, calculation for a non-diffusive Johnson-Segalman fluid with no material transport across the interface between phases. The dashed curve is the long-wave asymptotic form $\operatorname{Im}(\omega) \sim 0.1506 k^{2}$; the solid curve is the full numerical calculation. Points: (from highest to lowest) $l=0.00125,0.0025,0.005,0.01$. The figure on the left shows the true growth rate; on the right for the finite- $l$ results we have plotted $\operatorname{Im}(\omega)+10 l$.

\subsection{Comparison with results at small finite l; Fielding (2005)}

In this section we carry out a comparison with the numerical study of the full diffusive Johnson-Segalman model at $\Delta=0$, first published in [1]. From the findings of section 5.2, we expect that, for a given one-dimensional base state, the numerical results of [1] should converge, in the limit $l \rightarrow 0$, to the asymptotic results calculated here at $l=0$.

In figure 5, therefore, we reproduce (as points) the data from Figure 3 of [1] showing instability for small finite values of $l$, along with (as curves) numerical results for the pure, $l=0$ Johnson-Segalman fluid under the assumption that the interface is a material surface, and the long-wave asymptotic form for the same $l=0$ situation. On the left we present the raw data. From the numerical data we observe that $\operatorname{Im}(\omega) \sim a k^{2}+b(l)$ for long waves $k<1$, with $b(l) \approx-10 l$. We confirm the scaling of $b(l)$ with $l$ in the appendix. On the right, therefore, we add an additional term of $10 l$ to the numerical results for finite $l$, and observe that for long waves this collapses all the points onto the $l=0$ curve.

The numerical factor 10 which is used at order $l$ for the long-wave results is not calculated analytically, but deduced from the $k \rightarrow 0$ intercepts from the data for the different values of $l$. However, we can make some progress towards calculating this value. A full derivation of this process is given in appendix A: the final conclusion is that, as $k \rightarrow 0$ and $l \rightarrow 0$, the growth rate can be written as $\omega=i \sigma_{0} l+O\left(l^{2}\right)+O(k)$, where

$$
\sigma_{0} \sim \frac{-4\left[\bar{\mu}_{H} \kappa^{3}-\bar{\mu}_{L}(\kappa-1)^{3}\right] \bar{\mu}_{L} \bar{\mu}_{H}\left(\dot{\gamma}_{H}-\dot{\gamma}_{L}\right) \tilde{\sigma}}{\left[\bar{\mu}_{H} \kappa^{2}-\bar{\mu}_{L}(\kappa-1)^{2}\right]^{2}-4 \bar{\mu}_{L} \bar{\mu}_{H} \kappa(\kappa-1)}
$$




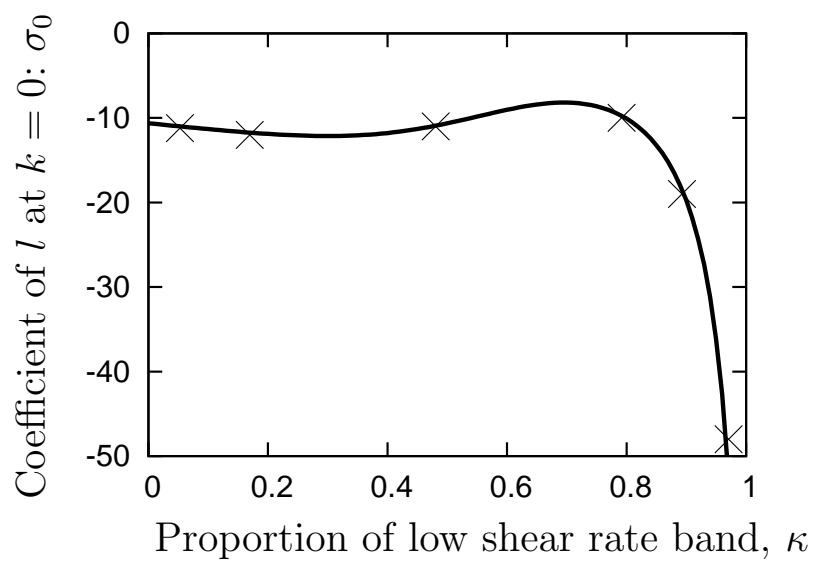

Fig. 6. Dependence of the coefficient of $l$ in the growth rate of very long-wave perturbations on the interface position, $\kappa$. Here $a=0.3, \epsilon=0.05$ and $T_{b}=0.506158$. The points are from numerical calculations and the curve is given by the analytical prediction from (86), with $\tilde{\sigma}$ chosen to match the data at $\kappa=0.79$.

and $\tilde{\sigma}$ is an unknown parameter which depends on $a, \epsilon$ and $T_{b}$ but not on $U_{\text {wall }}$ or $\kappa$. For the values of $a, \epsilon$ and $T_{b}$ associated with figure 5 the data suggest that $\tilde{\sigma} \approx 14$. In figure 6 we plot a numerical calculation of $\sigma_{0}$ against $\kappa$ for these values of $a, \epsilon$ and $T_{b}$. The curve is given by the prediction of (86) with $\tilde{\sigma}=14$. The agreement between theory and numerical calculation of this term is remarkable. As $\kappa \rightarrow 1$ our prediction (with $\tilde{\sigma}=14$ ) is $\sigma_{0} \approx-130$ : numerically it is not possible to investigate extremely narrow bands as the diffusive layer needs to be clear of the walls, but the numerical calculations of this long wave intercept match the theoretical prediction well even at $\kappa=0.97$ where $\sigma_{0} \approx-48$.

This diffusion-induced stability to very long waves $k \rightarrow 0$ was to be expected. Calculations had already shown that an interface between shear bands at this selected shear stress should be stable to one-dimensional perturbations: that is, if the whole interface is rigidly displaced from the selected position it should relax back there. A simple displacement of the interface corresponds to the limit of very long waves, and so this exponential relaxation is precisely the negative growth rate we have calculated in the limit $k \rightarrow 0$ with $l$ small but finite.

In figure 7 we show another comparison between the $l=0$ and $l \neq 0, \Delta=0$ cases, this time at $U_{\text {wall }}=4$. The convergence of the $l \neq 0$ results to the $l=0$ case as $l \rightarrow 0$ is clearly visible. At $l=0$ the growth rate for long waves is $\operatorname{Im}(\omega) \sim 0.42 k^{2}$, and the calculation of section 7.2 predicts $\sigma_{0}=-10.9$ for these parameters, so for long waves at small finite $l$ we expect $\operatorname{Im}(\omega) \sim$ $-10.9 l+0.42 k^{2}$, which means that instability first appears at $k \approx 5.1 l^{1 / 2}$. This scaling $k \sim l^{1 / 2}$ for the lowest unstable wavenumber is universal provided that long waves are unstable in the $l=0$ case. 


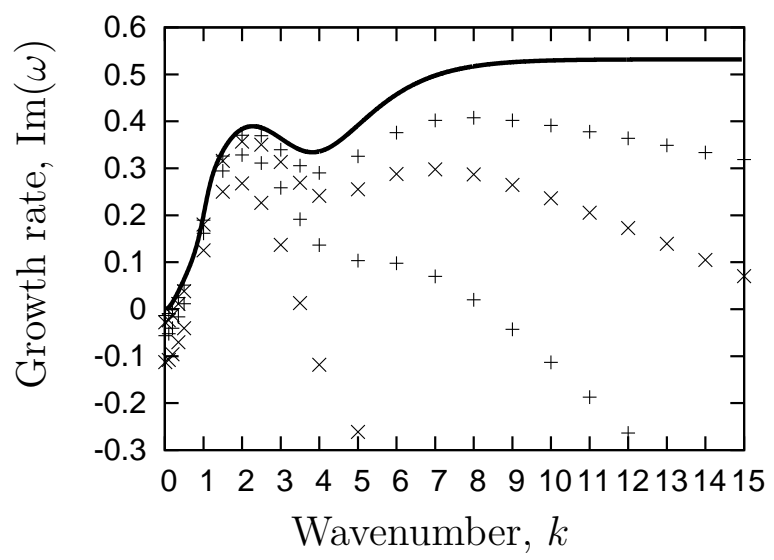

Fig. 7. Dependence of the growth rate of the instability on wavenumber. Parameters $\epsilon=0.05, a=0.3, T_{b}=0.506158, U_{\text {wall }}=4$. The long-wave mode leads to a peak growth rate at $k \approx 2$, but the growth rate increases again for shorter waves, and the most unstable mode (in the limit $l=0$ ) is for very short waves. The solid curve is for $l=0$; the points (from highest to lowest) are $l=0.00125,0.0025,0.005,0.01$.

For very short waves, we can see from figure 7 that the addition of diffusion has a large effect on the eigenvalue. We expected this when we stated in section 5 that our analysis would only be valid for $k \ll l^{-1}$. In fact we can see empirically that for these parameters, $\operatorname{Im}(\omega) \sim-12 k l+O(1)$ for fixed $l$ as $k \rightarrow \infty$, leading us to predict instability for $k \ll l^{-1}$ and stability for very large $k$. The size of the prefactor in this case means that the results for finite $l$ deviate from the $l=0$ limit earlier (as $k$ increases) than a simple scaling argument might have led us to expect.

In summary, if $0.108<\kappa<0.933$ (for the stress-diffusion model) then in the limit $l=0$ the flow is unstable to perturbations of all wavenumbers, and in this case we expect the diffusive flow to be unstable over a large region $l^{1 / 2} \ll k \ll l^{-1}$.

As a final comparison between our calculations and the numerics for small finite $l$, in figure 8 we give the perturbation streamfunction $\psi$ and its first derivative (proportional respectively to the cross- and along-channel velocity components) for one specific mode. We have plotted the real part of $\psi$ and its derivative at the phase ( $x$-position) where $\psi(\kappa)$ is real. The parameters (given in the caption to figure 8) are such that, in the limit $l=0$, long waves are unstable and the mode $k=0.1$ shows this growth. However, for $l=0.005$ the decay term at $O(l)$ dominates and the mode is stable. Nonetheless, the form of the streamfunction (equivalent to $10 i$ times the $y$-velocity in this case) and its first derivative (which is the $x$-velocity) is extremely similar between the two modes. 


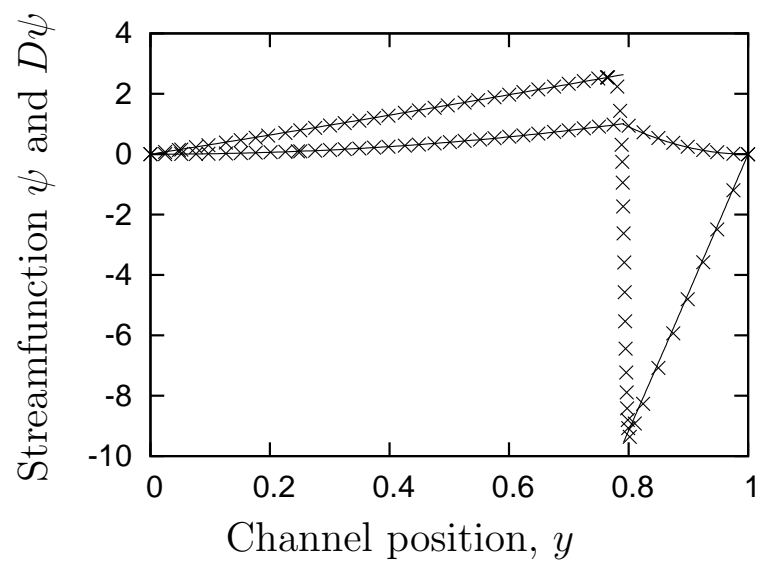

Fig. 8. The streamfunction $\psi$, proportional to $v$ (continuous function) and its derivative $D \psi$, or $u$ (discontinuous) of the least stable mode at $\epsilon=0.05, a=0.3, U_{\text {wall }}=2$, $T_{b}=0.506158, k=0.1$. This mode is unstable at $l=0$, with growth rate 0.00148 ; at $l=0.005$ it is stable with growth rate -0.0485454 . The crosses are from the full calculation at $l=0.005$, and the solid lines (lying under the crosses except near the interface) from the limit $l=0$ using the material surface condition. The streamfunction is plotted against position across the channel, $y$, and is normalised such that $\psi(\kappa)=1$. We show only the real part of $\psi$ and $D \psi$ here; the imaginary part is smaller by a factor of order $k$.

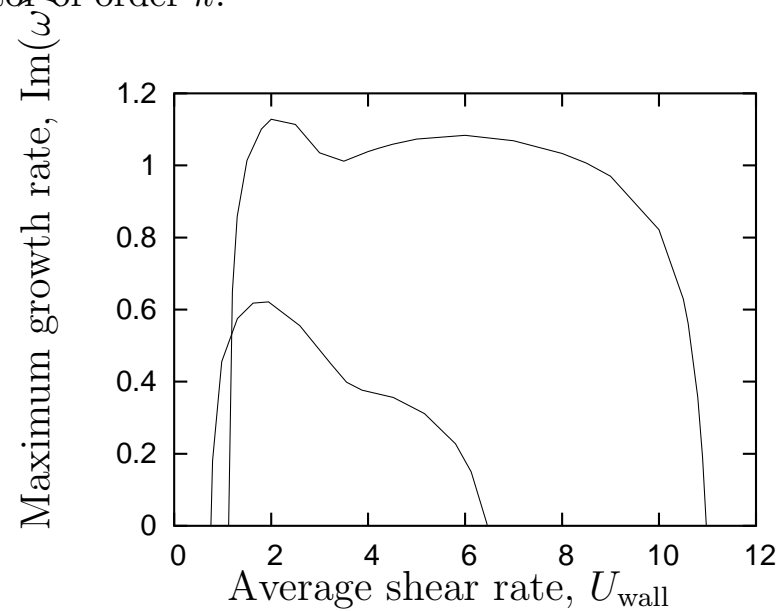

Fig. 9. Maximum growth rate plotted against average shear rate for a semi-empirical model of the stress-diffusion fluid with $l=0.00125, \epsilon=0.05$, and $\Delta=0$. Lower curve $a=0.3$; upper curve $a=0.8$. In both cases régimes with very narrow bands of one or other shear rate are stable to all perturbations. The wavenumber of the most unstable waves increases as we approach these stable regions.

\subsubsection{Most unstable mode with diffusion}

Finally, in figure 9 we give an empirical idea of the maximum growth rates which might be expected to be seen for a range of average shear rates, and for two different values of the slip parameter $a$. For the pure JS model, the most unstable mode is often the short-wave limit $k \rightarrow \infty$, which will be stabilised by diffusion for the modified model. It is therefore unhelpful to give the growth rate of the most unstable mode; rather, we attempt to give the expected most 
unstable growth rate in the presence of some diffusion $l=0.00125$. In order to carry out the computations for a large variety of parameters, we make two very broad assumptions:

- Based on curve fitting of the data in figure 7 , the growth rate at any $k$ and any a may be reasonably approximated by

$$
\operatorname{Im}(\omega)_{\text {approx }}=\operatorname{Im}(\omega)_{\mathrm{JS}}+\sigma_{0} l-13 k l .
$$

- In determining $\sigma_{0}$, the value $\tilde{\sigma}$ in equation (86), which is a function of $a$, $\epsilon$ and $T_{b}$, will be taken to be 14 (the true value at $a=0.3, \epsilon=0.05$ and $\left.T_{b}=0.505158\right)$ independent of these parameters.

Using these two assumptions and the numerical calculations for the pure JS problem, we predict the wavenumber and growth rate of the most unstable mode for two different fluids. We use the stress-diffusion model to select the shear stress in each case, and consider the cases $\epsilon=0.05, a=0.3$ and $\epsilon=0.05$, $a=0.8$.

There are configurations which are stable to all perturbations, which are those layer arrangements with one or other shear band being very narrow, i.e. $U_{\text {wall }} \approx \dot{\gamma}_{L}$ or $U_{\text {wall }} \approx \dot{\gamma}_{H}$. However, the vast majority of mean shear-rates in the shear banding régime produce banded flows which are linearly unstable with a moderate growth rate. The figure suggests that influence of the larger slip parameter $a=0.8$ tends to enhance the instability, but we cannot draw concrete conclusions from such an empirical model.

\subsection{Comparison with other previous studies}

\subsubsection{McLeish (1987)}

An early paper by McLeish [36] considered capillary flow with a constitutive equation with a non-monotonic flow curve. He predicted exactly the opposite of the long-wave behaviour we have found: for slow (i.e. long-wave) perturbations, he found instability only for very narrow regions of high-shear-rate material (the band close to the wall). That work used a slightly different constitutive equation [43], based on reptation theory for linear polymers, but his model has similar behaviour to ours in steady shear. He described the stability property of the flow as a dependence of the throughput in the lower-shear rate region on both the absolute position and the slope of the interface, with a formulation which is mathematically equivalent to ours except for the different base flow.

In terms of instability mechanism, McLeish suggests that since a normal stress 
difference is required in order for the system to "see" the gradient of the interface perturbation, normal stress effects are critical to the instability. From our calculations we see that this does appear to be true, but that the mechanism of instability is not quite the clean recirculation mechanism found by Hinch et al. [2] for coextruded fluids having matched viscosities and a jump in $N_{1}$ across the interface between them. In our equations there are two driving terms: the jump in $D \psi$, proportional to the difference in the base-state shear rate across the interface and the interface displacement (67); and the jump in $s_{12}$, proportional to the difference in the base-state $N_{1}$ across the interface and the slope of the interface (68). Algebraically, we can artificially separate these out, and in most cases studied here the normal stress term was weakly stabilising, and the instability comes from the interaction of the shear-rate-jump term with the normal stresses in the bulk of each fluid.

\subsubsection{Renardy (1995)}

Renardy [37] examined the stability of the local JS model in planar banded Couette flow. She found linear instability for short wavelengths (wavenumber greater than 8). For mainly historical reasons, however, she happened to confine her study to a base state corresponding to "top-jumping" $\left(T_{b}=T_{2}\right)$.

As a check on both our analysis and our numerical eigenvalue calculation, we reproduce figure 2 that paper. In Figure 10 we show our own numerical calculation, which duplicates her results as far as can be seen from the graph in [37]. In the second part of the figure we plot the same growth rate again, along with the long-wave asymptotic form for the growth rate as it depends on wavenumber. We have restricted the scale in this second graph in order to better see the accuracy of the long-wave result.

Since neither of the two non-local models predicts the top-jumping stress $T_{2}$ as the selected stress, this work is unlikely to be directly relevant physically; moreover, the bulk of [37] focuses on a short-wave instability. While this instability does occur for the original JS model systems at the true stress selected by a nonlocal model (for instance, at $\epsilon=0.05$ and $a=0.3$ using $T_{b}$ as selected by stress diffusion, it has growth rate 0.533 ), when diffusion is added the short-wave instability mechanism is destroyed and this mode will not be seen.

\subsubsection{Yuan (1999)}

In 1999, Yuan [39] carried out time-dependent simulations using a model corresponding to $\Delta=1$ in our model. He found that, for $\epsilon=0.05$ and $a=0.8$, the system uniquely selected a shear stress of $T_{b}=0.81 \pm 0.04$ for any average shear rate in the range over which a homogeneous solution would be on 

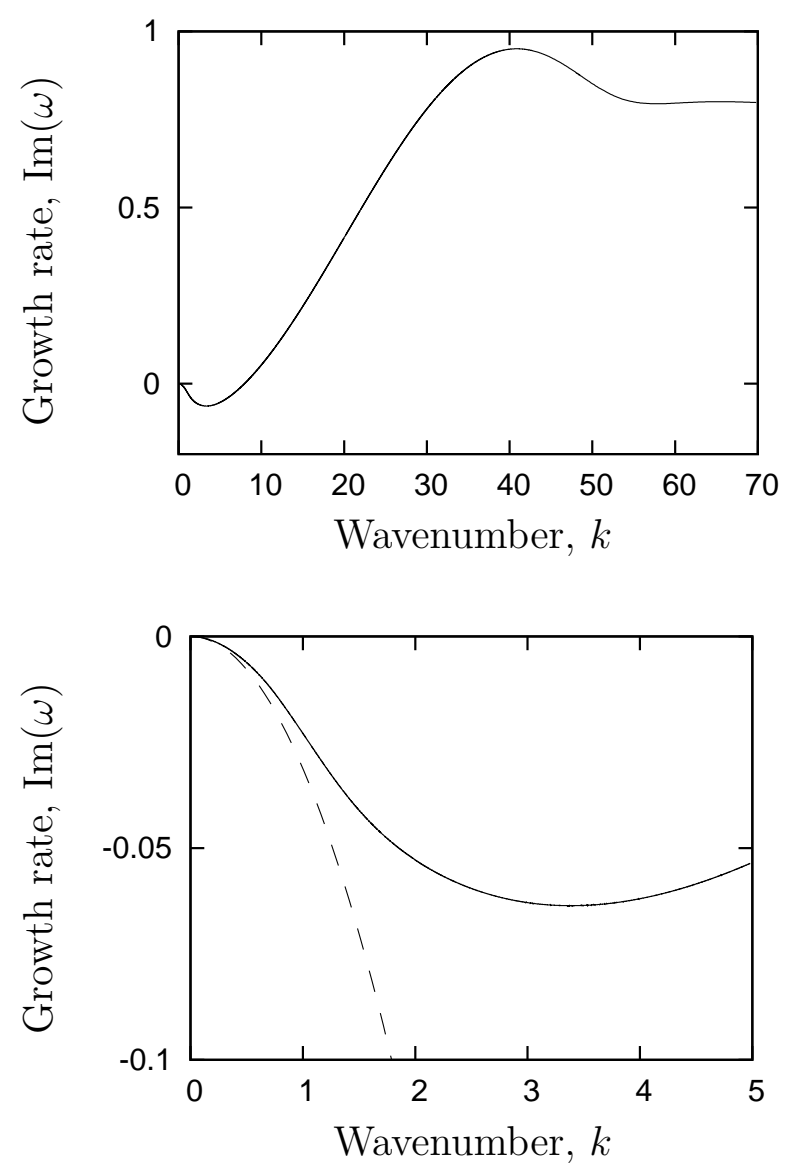

Fig. 10. Reproduction of figure 2 from Renardy [37]. Parameters are $\epsilon=0.05$, $a=0.8, T_{b}=0.921$. Growth rate is plotted against wavenumber. The upper figure reproduces the original exactly (to the naked eye; the original data were not available) and in the lower figure we reproduce the long-wave portion of the graph. Solid curve: numerical calculations; dotted line: asymptote $-0.0312 k^{2}$ from long wave calculation. As stated in the text of [37], instability appears for waves shorter than $k \approx 8$, and the peak growth rate of instability is for waves having $k \approx 41$.

the unphysical descending branch of the constitutive curve. This gives values $T_{b}^{*}=\left(1-a^{2}\right)^{1 / 2} T_{b}=0.486 \pm 0.024$, which is slightly outside the true value of 0.45 from equation (31).

He found stable steady states for all parameters in this range. Our longwave analysis predicts instability to long waves at his parameter values for $0.186<\kappa<0.974$, that is, $1.13<U_{\text {wall }}<7.56$, suggesting that several of his simulated flows (at $U_{\text {wall }}=2,3,4,5$ and 6 ) should be unstable to long-wave perturbations. However, he was simulating a finite length of channel (and with a relatively large value of $l$ ) which allows access only to specific wavenumbers, which may be the reason that he did not capture the instability we have demonstrated. Alternatively, Yuan's results may be an artefact of averaging over time $t$ and/or flow location, $x$. Note that if these simulations are timeaverages of a nonlinearly fluctuating interface where a flat interface is linearly 
unstable, then we would expect the average shear stress reported to be higher than the selected value for the 1D base state, as observed above.

Another comment which seems unusual is that Yuan states that even when $l=0$, the interface has finite width. Although there are physical reasons why this may be true in practice, it is not predicted by the governing equations in the limit $l=0$ : perhaps his observation is a grid-scale effect.

\section{Conclusions and Discussion}

We have investigated the two-dimensional linear stability of plane Couette flow of a shear-banding fluid. If the equations governing the Johnson-Segalman model are regularised using a small amount of stress diffusion to provide a uniquely selected one-dimensional banded base state, we have shown that as far as two-dimensional linear stability is concerned, the limit of no diffusion is a regular limit in which the interface region becomes a strict material interface.

Using this limit, we have demonstrated that there is a long wave instability for almost all possible positions of a shear band. Only the case of a very narrow "spurted" region of high shear rate is stable to long-wave perturbations. This is in contrast to earlier work by McLeish [36], who found (for Poiseuille flow and slightly different constitutive assumptions) stability except for the case of a narrow high-shear rate band; and simulations by Yuan [39], which predict a steady interface between two shear bands in this situation. However, we agree quantitatively with results of Renardy [37], who happened to look at a narrow region of high shear and found stability to long waves (although the paper focuses on a short-wave instability whose mechanism is likely to be affected by diffusion). Our results are in full agreement with numerical stability calculations including diffusion terms [1]. For typical physical parameters, for which there is instability to perturbations of all wavelengths in the absence of diffusion, we have identified the scalings at which diffusion affects the instability. Small diffusion on a dimensionless lengthscale $l$ will restabilise very long and very short waves, leaving the flow unstable to perturbations of moderate dimensionless wavenumber $l^{1 / 2}<k \ll 1^{-1}$.

We have identified two driving forces for the instability: discontinuity of shear rate and of normal stress across an interface. The interplay between these mechanisms, even for long waves, is not fully understood.

This widespread instability suggests that the existing theoretical picture of two stable shear bands separated by a steady interface needs further thought. Indeed, this result is consistent with accumulating evidence for erratic fluctuations $[11,9,12,44,45]$ in several different shear banding systems. 
Future work will investigate the behaviour of the interface in the nonlinear régime, beyond the validity of this linear study. One possibility is that the instability saturates at a small but finite amplitude — indeed, our preliminary investigations suggest that this is the case. This would be consistent with a narrowly localised but still unsteady interface, which might be interpreted as steady in experiments that did not have high spatial resolutions. This might even reconcile early data showing apparently steady interfaces with recent work revealing fluctuations.

However, if this is not the case, then the use of a Johnson-Segalman type constitutive model, with or without stress diffusion, can almost never produce agreement with any steady banded structure observed experimentally. One would then need a new theoretical picture to incorporate the observed shearbanding effects within a stable flow which is either steady or undergoes only small-amplitude oscillations.

\section{Acknowledgements}

The authors would like to thank the referees for their helpful comments.

\section{A Calculation of the order $l$ contribution to $\omega$ in the long-wave limit}

In this section we derive a scaling form for the (negative) growth rate of a perturbation at $k=0$ for small $l$, in the case $\Delta=0$, i.e. the case in which the stress diffusion is added through diffusion of the polymer extra stress term. This negative growth appears at order $l$.

Let us return to the full governing equations (37)-(43). We will first scale with $k$ and then with $l$.

Using the long-wave scalings of section 6 , but allowing $\omega$ to remain order 1 (with respect to $k$ ), and neglecting terms of order $k$ yields the system

$$
\begin{gathered}
i \bar{s}_{11}+D s_{12}=0 \quad D \bar{s}_{22}=0 \\
\bar{s}_{11}=\bar{s}_{22}=-\bar{p} \quad s_{12}=\epsilon D^{2} \psi+t_{12} \\
(-i \omega+1) t_{11}=l^{2} D^{2} t_{11}+\left(1-a^{2}\right) \dot{\gamma} t_{12}+\left(1-a^{2}\right) T_{12} D^{2} \psi \\
(-i \omega+1) t_{12}=l^{2} D^{2} t_{12}-\dot{\gamma} t_{11}+\left(1-T_{11}\right) D^{2} \psi
\end{gathered}
$$


As before, we solve the momentum equations to have

$$
s_{12}=A y+B \quad \bar{s}_{11}=\bar{s}_{22}=-\bar{p}=i A .
$$

We also scale the eigenvalue: $\omega=i l \sigma_{0}+O\left(l^{2}\right)$.

We now divide the flow into three regions: the two "outer" regions where the base state shear rate and stresses are constant, and the "inner" region where base state quantities have derivatives of order $l^{-1}$. We denote by $X_{L}$ the value of a quantity in the low-shear-rate band near the wall at $y=0$, and by $X_{H}$ its value in the high-shear-rate band near the wall at $y=1$. As in section 6 , we will use the marginal viscosity

$$
\bar{\mu}=\epsilon+\frac{\left(1-2 T_{11}\right)}{\left(1+\left(1-a^{2}\right) \dot{\gamma}^{2}\right)} .
$$

In each outer region, where derivatives are order 1 , we neglect terms of order $l$ and solve to have

$$
\begin{array}{cc}
t_{12}^{L}=\frac{[A y+B]\left(\bar{\mu}_{L}-\epsilon\right)}{\bar{\mu}_{L}} & t_{11}^{L}=\frac{2[A y+B]\left(1-a^{2}\right) T_{12}^{L}}{\bar{\mu}_{L}\left[1+\left(1-a^{2}\right) \dot{\gamma}_{L}^{2}\right]} \\
D^{2} \psi_{L}=[A y+B] / \bar{\mu}_{L} & \psi_{L}=\left[A y^{3}+3 B y^{2}\right] / 6 \bar{\mu}_{L}
\end{array}
$$

in the low-shear band, and in the high-shear band,

$$
\begin{gathered}
t_{12}^{H}=\frac{[A y+B]\left(\bar{\mu}_{H}-\epsilon\right)}{\bar{\mu}_{H}} \quad t_{11}^{H}=\frac{2[A y+B]\left(1-a^{2}\right) T_{12}^{H}}{\bar{\mu}_{H}\left[1+\left(1-a^{2}\right) \dot{\gamma}_{H}^{2}\right]} \\
D^{2} \psi_{H}=[A y+B] / \bar{\mu}_{H} \quad \psi_{H}=\left[A\left(y^{3}-3 y+2\right)+3 B(y-1)^{2}\right] / 6 \bar{\mu}_{H} .
\end{gathered}
$$

Within the inner region, we scale lengths as $\xi=(y-\kappa) / l$ so that $l D=d$ and pose the series

$$
t_{i j} \sim l^{-1} t_{i j}^{-1}+t_{i j}^{0}+\cdots \quad \psi \sim \psi^{0}+l \psi^{1}+l^{2} \psi^{2}+\cdots
$$

Matching these quantities to the outer solutions for large $|\xi|$ yields the following conditions:

$$
\begin{gathered}
t_{i j}^{-1} \rightarrow 0, d \psi^{0} \rightarrow 0, d^{2} \psi^{1} \rightarrow 0 \text { as } \xi \rightarrow \pm \infty \\
t_{i j}^{0} \rightarrow t_{i j}^{L}(\kappa), \psi^{0} \rightarrow \psi_{L}(\kappa), d \psi^{1} \rightarrow D \psi_{L}(\kappa), d^{2} \psi^{2} \rightarrow D^{2} \psi_{L}(\kappa)
\end{gathered}
$$

as $\xi \rightarrow-\infty$; and as $\xi \rightarrow \infty$,

$$
t_{i j}^{0} \rightarrow t_{i j}^{H}(\kappa), \psi^{0} \rightarrow \psi_{H}(\kappa), d \psi^{1} \rightarrow D \psi_{H}(\kappa), d^{2} \psi^{2} \rightarrow D^{2} \psi_{H}(\kappa)
$$


We collect orders of $l$ in the resulting equations. At order $l^{-2}$ we have simply

$$
d^{2} \psi^{0}=0
$$

which, along with (A.12) gives $\psi^{0}=\alpha_{0}$. At order $l^{-1}$ we have

$$
\begin{gathered}
\epsilon d^{2} \psi^{1}+t_{12}^{-1}=0 \\
d^{2} t_{11}^{-1}-t_{11}^{-1}+\left(1-a^{2}\right) \dot{\gamma} t_{12}^{-1}+\left(1-a^{2}\right) T_{12} d^{2} \psi^{1}=0 \\
d^{2} t_{12}^{-1}-t_{12}^{-1}-\dot{\gamma} t_{11}^{-1}+\left(1-T_{11}\right) d^{2} \psi^{1}=0
\end{gathered}
$$

which are solved by

$$
t_{11}^{-1}=-\delta d T_{11} \quad t_{12}^{-1}=-\delta d T_{12} \quad d^{2} \psi^{1}=-\delta d \dot{\gamma}
$$

Substituting the matching conditions from (A.13)-(A.14) and the outer solutions leads to the condition

$$
A \kappa+B=\frac{4 \delta\left[\bar{\mu}_{H} \kappa^{3}-\bar{\mu}_{L}(\kappa-1)^{3}\right] \bar{\mu}_{L} \bar{\mu}_{H}\left(\dot{\gamma}_{H}-\dot{\gamma}_{L}\right)}{\left[\bar{\mu}_{H} \kappa^{2}-\bar{\mu}_{L}(\kappa-1)^{2}\right]^{2}-4 \bar{\mu}_{L} \bar{\mu}_{H} \kappa(\kappa-1)} .
$$

Finally, at order 1 our remaining equations are

$$
\begin{gathered}
\epsilon d^{2} \psi^{2}+t_{12}^{0}=A \kappa+B \\
d^{2} t_{11}^{0}-t_{11}^{0}+\left(1-a^{2}\right) \dot{\gamma} t_{12}^{0}+\left(1-a^{2}\right) T_{12} d^{2} \psi^{2}=-\sigma_{0} \delta d T_{11} \\
d^{2} t_{12}^{0}-t_{12}^{0}-\dot{\gamma} t_{11}^{0}+\left(1-T_{11}\right) d^{2} \psi^{2}=-\sigma_{0} \delta d T_{12}
\end{gathered}
$$

Where equations (A.16)-(A.18) were homogeneous, these are inhomogeneous ODEs with a forcing on the RHS which comes from the previous order calculation. Together with the conditions on $t_{i j}^{0}$ from (A.13) and (A.14), they provide a constraint on $\sigma_{0}$.

Although we cannot solve this problem, we can reduce its complexity. If we define

$$
\alpha=d^{2} \psi^{2} /[A \kappa+B] \quad \tilde{t}_{i j}=t_{i j}^{0} /[A \kappa+B] \quad \tilde{\sigma}=-\sigma_{0} \delta /[A \kappa+B]
$$

then the governing equations become

$$
\begin{gathered}
\epsilon \alpha+\tilde{t}_{12}=1 \\
d^{2} \tilde{t}_{11}-\tilde{t}_{11}+\left(1-a^{2}\right) \dot{\gamma} \tilde{t}_{12}+\left(1-a^{2}\right) T_{12} \alpha=\tilde{\sigma} d T_{11} \\
d^{2} \tilde{t}_{12}-\tilde{t}_{12}-\dot{\gamma} \tilde{t}_{11}+\left(1-T_{11}\right) \alpha=\tilde{\sigma} d T_{12}
\end{gathered}
$$

and the matching conditions,

$$
\tilde{t}_{11} \rightarrow \frac{2\left(1-a^{2}\right) T_{12}^{L}}{\bar{\mu}_{L}\left[1+\left(1-a^{2}\right) \dot{\gamma}_{L}^{2}\right]} \quad \tilde{t}_{12} \rightarrow \frac{\bar{\mu}_{L}-\epsilon}{\bar{\mu}_{L}} \text { as } \xi \rightarrow-\infty
$$




$$
\tilde{t}_{11} \rightarrow \frac{2\left(1-a^{2}\right) T_{12}^{H}}{\bar{\mu}_{H}\left[1+\left(1-a^{2}\right) \dot{\gamma}_{H}^{2}\right]} \quad \tilde{t}_{12} \rightarrow \frac{\bar{\mu}_{H}-\epsilon}{\bar{\mu}_{H}} \text { as } \xi \rightarrow \infty .
$$

Neither the governing equations nor the matching conditions have any dependence on $\kappa$, the position of the interface between the two shear rate bands. Thus there is a single value of $\tilde{\sigma}$ for each set of parameters $\left\{a, \epsilon, T_{b}\right\}$ independent of $U_{\text {wall }}$ and $\kappa$. The long-wave term of the eigenvalue $\omega$ is then given by

$$
\omega \sim \frac{-4 i l\left[\bar{\mu}_{H} \kappa^{3}-\bar{\mu}_{L}(\kappa-1)^{3}\right] \bar{\mu}_{L} \bar{\mu}_{H}\left(\dot{\gamma}_{H}-\dot{\gamma}_{L}\right) \tilde{\sigma}}{\left[\bar{\mu}_{H} \kappa^{2}-\bar{\mu}_{L}(\kappa-1)^{2}\right]^{2}-4 \bar{\mu}_{L} \bar{\mu}_{H} \kappa(\kappa-1)}+O\left(l^{2}\right)+O(k)
$$

For the results given in figure 3 of [1], we had $a=0.3, \epsilon=0.05$ and $T_{b}=$ 0.506158 , giving shear rates of $\dot{\gamma}_{L}=0.66143$ and $\dot{\gamma}_{H}=7.0893$. The interface position was 0.79176 and a fit of the data gave $\omega \sim-10 l$. We can deduce that

$$
\tilde{\sigma}\left(a=0.03, \epsilon=0.05, T_{b}=0.506158\right) \approx 14 .
$$

\section{References}

[1] S. M. Fielding, Linear instability of planar shear banded flow, Phys. Rev. Lett. 95 (2005) 134501.

[2] E. J. Hinch, O. J. Harris, J. M. Rallison, The instability mechanism for two elastic liquids being coextruded, J. Non-Newt. Fluid Mech. 43 (1992) 311-324.

[3] M. M. Britton, P. T. Callaghan, Two-phase shear band structures at uniform stress, Phys. Rev. Lett. 78 (26) (1997) 4930-4933.

[4] O. Diat, D. Roux, F. Nallet, Effect of shear on a lyotropic lamellar phase, J. de Physique II 3 (1993) 1427-1452.

[5] E. Cappelaere, R. Cressely, J. P. Decruppe, Linear and nonlinear rheological behavior of salt-free aqueous CTAB solutions, Colloids Surf. A 104 (1995) 353374.

[6] R. W. Mair, P. T. Callaghan, Observation of shear banding in worm-like micelles by NMR velocity imaging, Europhys. Lett. 36 (1996) 719-724.

[7] L. Bécu, S. Manneville, A. Colin, Spatiotemporal dynamics of wormlike micelles under shear, Phys. Rev. Lett. 93 (2004) 018301.

[8] J. S. Raynaud, P. Moucheront, J. C. Baudez, F. Bertrand, J. P. Guilbaud, P. Coussot, Direct determination by nuclear magnetic resonance of the thixotropic and yielding behavior of suspensions, J. Rheol. 46 (3) (2002) 709732 . 
[9] R. Bandyopadhyay, G. Basappa, A. K. Sood, Observation of chaotic dynamics in dilute sheared aqueous solutions of CTAT, Phys. Rev. Lett. 84 (9) (2000) 2022-2025.

[10] E. K. Wheeler, P. Fischer, G. G. Fuller, Time-periodic flow induced structures and instabilities in a viscoelastic surfactant solution, J. Non-Newt. Fluid Mech. 75 (1998) 193-208.

[11] A. S. Wunenburger, A. Colin, J. Leng, A. Arneodo, D. Roux, Oscillating viscosity in a lyotropic lamellar phase under shear flow, Phys. Rev. Lett. 86 (7) (2001) 1374-1377.

[12] W. M. Holmes, M. R. López-González, P. T. Callaghan, Fluctuations in shearbanded flow seen by NMR velocimetry, Europhys. Lett. 64 (2) (2003) 274-280.

[13] M. R. López-González, W. M. Holmes, P. T. Callaghan, P. J. Photinos, Shear banding fluctuations and nematic order in wormlike micelles, Phys. Rev. Lett. 93 (2004) 268302.

[14] S. Manneville, J. B. Salmon, A. Colin, A spatio-temporal study of rheooscillations in a sheared lamellar phase using ultrasound, Eur. Phys. J. E 13 (2004) 197-212.

[15] J. B. Salmon, S. Manneville, A. Colin, Shear banding in a lyotropic lamellar phase. II. temporal fluctuations, Phys. Rev. E 68 (2003) 051504.

[16] N. A. Spenley, M. E. Cates, T. C. B. McLeish, Nonlinear rheology of wormlike micelles, Phys. Rev. Lett. 71 (1993) 939-942.

[17] R. Makhloufi, J. Decruppe, A. Ait-Ali, R. Cressely, Rheo-optical study of wormlike micelles undergoing a shear banding flow, Europhys. Lett. 32 (1995) 253258.

[18] R. W. Mair, P. T. Callaghan, Shear flow of wormlike micelles in pipe and cylindrical couette geometries as studied by nuclear magnetic resonance microscopy, J. Rheol. 41 (1997) 901-924.

[19] M. Johnson, D. Segalman, A model for viscoelastic fluid behaviour which allows non-affine deformation, J. Non-Newt. Fluid Mech. 2 (1977) 255-270.

[20] J. Yerushalmi, S. Katz, R. Shinnar, The stability of steady shear flows of some viscoelastic fluids, Chem. Engg Sci. 25 (1970) 1891-1902.

[21] N. A. Spenley, X.-F. Yuan, M. E. Cates, Nonmonotonic constitutive laws and the formation of shear-banded flows, J. de Physique II 6 (1996) 551-571.

[22] P. D. Olmsted, O. Radulescu, C.-Y. D. Lu, The Johnson-Segalman model with a diffusion term in cylindrical Couette flow, J. Rheol. 44 (2000) 257-275.

[23] D. S. Malkus, J. S. Nohel, B. J. Plohr, Dynamics of shear flow of a nonNewtonian fluid, J. Comp. Phys. 87 (1990) 464-487.

[24] D. S. Malkus, J. S. Nohel, B. J. Plohr, Analysis of new phenomena in shear flow of non-Newtonian fluids, SIAM J. Appl. Math. 51 (1991) 899-929. 
[25] C.-Y. D. Lu, P. D. Olmsted, R. C. Ball, The effect of non-local stress on the determination of shear banding flow, Phys. Rev. Lett. 84 (2000) 642-645.

[26] P. D. Olmsted, C.-Y. D. Lu, Coexistence and phase separation in sheared complex fluids, Phys. Rev. E56 (1997) 55-58.

[27] P. D. Olmsted, P. M. Goldbart, Isotropic-nematic transition in shear flow: State selection, coexistence, phase transitions, and critical behavior, Phys. Rev. A46 (1992) 4966-4993.

[28] A. W. El-Kareh, L. G. Leal, Existence of solutions for all Deborah numbers for a non-Newtonian model modified to include diffusion, J. Non-Newt. Fluid Mech. 33 (1989) 257-287.

[29] O. Radulescu, P. D. Olmsted, Matched asymptotic solutions for the steady banded flow of the diffusive Johnson-Segalman model in various geometries, J. Non-Newt. Fluid Mech. 91 (2000) 143-164.

[30] P. D. Olmsted, O. Radulescu, C.-Y. D. Lu, Shear-banding in the diffusive Johnson-Segalman model, in: XIIIth International Congress on Rheology, Vol. 3, 2000, pp. 228-230.

[31] X.-F. Yuan, Dynamics of a mechanical interface in shear-banded flow, Europhys. Lett. 46 (1999) 542-548.

[32] P. Español, X.-F. Yuan, R. Ball, Shear banding in the Johnson-Segalman fluid, J. Non-Newt. Fluid Mech. 65 (1996) 93-109.

[33] G. C. Georgiou, D. Vlassopoulos, On the stability of the simple shear flow of a Johnson-Segalman fluid, J. Non-Newt. Fluid Mech. 75 (1998) 77-97.

[34] S. M. Fielding, P. D. Olmsted, Spatiotemporal oscillations and rheochaos in a simple model of shear banding, Phys. Rev. Lett. 92 (2004) 084502.

[35] A. Aradian, M. E. Cates, Instability and spatiotemporal rheochaos in a shearthickening fluid model, Europhys. Lett. 70 (3) (2005) 397-403.

[36] T. C. B. McLeish, Stability of the interface between two dynamic phases in capillary flow of linear polymer melts, J. Poly. Sci. B: Poly. Phys. 25 (1987) 2253-2264.

[37] Y. Y. Renardy, Spurt and instability in a two-layer Johnson-Segalman liquid, Theoret. Comput. Fluid Dynamics 7 (1995) 463-475.

[38] S. M. Fielding, P. D. Olmsted, Nonlinear dynamics of an interface between shear bands, Phys. Rev. Lett. 96 (2006) 104502.

[39] X.-F. Yuan, Interfacial dynamics of viscoelastic fluid flows, Phys. Chem. Chem. Phys. 1 (1999) 2177-2182.

[40] R. Kupferman, On the linear stability of plane Couette flow for an Oldroyd-B fluid and its numerical approximation, J. Non-Newt. Fluid Mech. 127 (2005) 169-190. 
[41] S. M. Fielding, P. D. Olmsted, Kinetics of the shear banding instability in startup flows, Phys. Rev. E 68 (2003) 036313.

[42] T. C. Ho, M. M. Denn, Stability of plane Poiseuille flow of a highly elastic liquid, J. Non-Newt. Fluid Mech. 3 (2) (1977/78) 179-195.

[43] T. C. B. McLeish, R. C. Ball, A molecular approach to the spurt effect in polymer melt flow, J. Poly. Sci. B: Poly. Phys. 24 (1986) 1735-1745.

[44] Y. T. Hu, P. Boltenhagen, D. J. Pine, Shear thickening in low-concentration solutions of wormlike micelles. I. Direct visualization of transient behavior and phase transitions, J. Rheol. 42 (5) (1998) 1185-1208.

[45] P. Fischer, E. K. Wheeler, G. G. Fuller, Shear-banding structure orientated in the vorticity direction observed for equimolar micellar solution, Rheol. Acta $41(1-2)(2002) 35-44$. 